\title{
Quels liens entre tension sur le marché du travail, difficultés de recrutement et efforts de recherche des employeurs?
}

What are the Links between Labour Market Tightness, Recruitment Difficulties, and Employers' Search Efforts?

\section{Bertrand Lhommeau et Véronique Rémy}

\section{OpenEdition \\ Journals}

Édition électronique

URL : https://journals.openedition.org/travailemploi/10579

ISSN : 1775-416X

Éditeur

DARES - Ministère du Travail

Édition imprimée

Date de publication : 15 décembre 2020

Pagination : 45-82

ISSN : 0224-4365

Référence électronique

Bertrand Lhommeau et Véronique Rémy, «Quels liens entre tension sur le marché du travail, difficultés de recrutement et efforts de recherche des employeurs? », Travail et Emploi [En ligne], 163 | 2020, mis en ligne le 01 février 2022, consulté le 26 février 2022. URL : http:// journals.openedition.org/travailemploi/10579 


\title{
Quels liens entre tension sur le marché du travail, difficultés de recrutement et efforts de recherche des employeurs?
}

\author{
Bertrand Lhommeau ${ }^{*}$, Véronique Rémy ${ }^{* *}$
}

Cet article étudie les liens entre tension sur le marché du travail, difficultés de recrutement et moyens mobilisés par les recruteurs à l'aide de l'enquête Offre d'emploi et recrutement (Ofer) 2016 de la Direction de l'animation de la recherche, des études et des statistiques (Dares). La construction d'une typologie selon l'intensité de la tension, les difficultés de recrutements anticipées, la concurrence de la part des autres employeurs et le nombre de demandeurs d'emploi disponibles permet d'identifier cinq classes de métiers dont deux associent tension et difficultés anticipées élevées. Cette typologie permet d'analyser les difficultés d'embauche déclarées ex post et les efforts de recrutement des employeurs. Recruter sur un métier tendu n'est pas systématiquement plus difficile, mais s'accompagne plus souvent d'un déficit d'image ou de candidatures. Concernant les relations entre tension et efforts de recrutement, recruter sur des métiers en tension n'implique pas l'activation de davantage de canaux de recherche de candidats, mais davantage de demandes d'éléments aux candidats et d'entretiens individuels avec ces derniers.

$\mathrm{D}$ ans le débat public, l'idée selon laquelle, malgré un nombre de chômeurs élevé, certains employeurs rencontrent des difficultés pour pourvoir leurs postes vacants est récurrente. «Emplois vacants », « tension sur le marché du travail ${ }^{1}$ » et « difficultés de recrutement » sont alors souvent associés, parfois de manière peu distincte, pour décrire ce qui s'apparente à un paradoxe. De leur côté, les employeurs pointent souvent comme source de difficultés l'inadéquation du profil des candidats au poste

\footnotetext{
* Au moment de la rédaction de cet article, Département analyse des métiers et des travailleurs handicapés, Dares, ministère du Travail, de l'Emploi et de l'Insertion. Actuellement, Insee; bertrand.lhommeau@insee.fr.

** Mission animation de la recherche, Dares, ministère du Travail, de l'Emploi et de 1'Insertion; veronique.remy @ travail.gouv.fr.

1. Définie comme le ratio entre nombre d'emplois vacants et nombre de personnes en recherche d'emploi.
} 
proposé (BLAChe, Buchner, 2015; JAUdEAU et al., 2015; InSEe, 2017; LHOMMEAU, RÉMY, 2019a). Mais l'inexpérience des employeurs en matière de recrutement, ou l'insuffisance des moyens affectés à cette activité pourraient également être en cause dans les difficultés qu'ils rencontrent. C'est l'hypothèse que nous formulons ici, en interrogeant les liens entre trois dimensions : le contexte du marché du travail, l'effort de recrutement des employeurs et les difficultés rencontrées lors de l'embauche.

Que sait-on des liens entre contexte du marché du travail et difficultés de recrutement, premier volet de notre triptyque ? Un nombre élevé d'emplois à pourvoir à une date donnée est souvent considéré comme le signe de difficultés de recrutement. Or c'est loin d'être toujours le cas (Fondeur, ZANDA², 2009; CoE, 2013; Estrade, 2013), notamment parce que le processus de rencontre entre offreurs et demandeurs d'emploi - processus d'appariement - prend naturellement du temps, les parties en présence devant acquérir de l'information l'une sur l'autre. Des personnes en recherche d'emploi et des postes vacants peuvent ainsi coexister sur le marché du travail générant du chômage « frictionnel» (PISSARIDES, 2000). Seuls les postes durablement vacants ou les abandons de recrutement - évalués par Pôle emploi à 255000 au $1^{\text {er }}$ trimestre de 2015 sur le champ des offres qui y sont déposées ${ }^{3}$ - sont le signe de véritables difficultés de recrutement. De plus, parmi les abandons, ceux correspondant à une disparition du besoin de recruter ne reflètent pas la présence d'obstacles à l'embauche. Si de nombreuses sources - enquêtes Besoins en main-d'œuvre (BMO) de Pôle emploi, Tendance, emploi, compétence (TEC) du Medef ${ }^{4}$, enquêtes de conjoncture de l'Insee ${ }^{5}$ et de la Banque de France - existent pour mesurer l'ampleur des difficultés de recrutement et leur nature du point de vue des employeurs, les travaux analysant l'impact du contexte du marché du travail sur ces difficultés sont rares.

La durée à prendre en compte pour considérer qu' un recrutement est difficile (COE, 2013) et la nature des difficultés rencontrées lors de l'embauche diffèrent fortement selon le métier considéré : ce dernier nécessite-t-il des compétences très spécifiques ? Celles-ci sont-elles répandues parmi les personnes en recherche d'emploi, notamment sur le marché du travail local? Sont-elles très recherchées par d'autres employeurs? Le contexte dans lequel l'employeur recrute est donc susceptible de varier selon la nature du poste à pourvoir: en 2019, les employeurs anticipent davantage de difficultés de recrutement lorsqu'ils embauchent sur un poste d'ingénieur informaticien, un métier pour lequel la tension est élevée, que sur un poste de secrétaire pour lequel cette dernière est plus faible (NIANG, VROYLANDT, 2020). Le métier constitue ainsi une maille particulièrement pertinente pour étudier la correspondance entre l'offre et la demande d'emploi et les éventuelles tensions qui en résultent. En effet, ce niveau

2. Comme mentionné par ces auteurs, « une fois écarté le risque de confondre emplois vacants et difficultés de recrutement, il ne faudrait pas oublier que ce sont ces dernières qu'il convient d'analyser en priorité » (p. 4).

3. Plus précisément, il s'agit des offres d'emploi satisfaites en plus de quatre semaines et des offres annulées par

l'employeur après quinze jours de dépôt (BLACHE et al., 2016).

4. Mouvement des entreprises de France.

5. Institut national de la statistique et des études économiques. 
d'analyse permet de confronter, d'une part, les tâches associées par l'employeur au poste à pourvoir et, d'autre part, les compétences détenues par les candidats à ce poste, repérables notamment par la nature de leur formation ou leur expérience. Les méthodes de recrutement diffèrent également notablement selon les métiers, en lien avec leur secteur d'activité. Ainsi, les canaux de recherche de candidats que l'employeur active - annonces, réseaux de relations, Pôle emploi, etc. - dépendent du segment du marché du travail sur lequel il se place : dans le secteur de l'hôtellerie-restauration où le recrutement a souvent lieu dans l'urgence, les recruteurs font davantage appel aux candidatures spontanées ou à leur réseau tandis que pour pourvoir les postes d'employés dans la grande distribution, ils favorisent les candidatures spontanées et les annonces dans leurs locaux (Forté, Monchatre, 2013; Rieucau, Salognon, 2013). De leur côté, les sociétés de service informatique privilégient la diffusion d'offres d'emploi en ligne pour embaucher sur des postes d'ingénieurs informatiques (FonDEUR, 2013). Les autres étapes du processus du recrutement diffèrent également selon le métier du poste à pourvoir : par exemple, les compétences personnelles, plus souvent recherchées lors des embauches sur des métiers de services - et a fortiori de contact avec le public - ne sont pas appréciées à l'aide des mêmes méthodes de sélection que la détention d'un diplôme, facilement repérable dans un curriculum vitae (CV) (BAILLY, LÉNÉ, 2015).

Deuxième volet de notre triptyque: les employeurs ajustent-ils leurs efforts de recherche et de sélection des candidats pour faire face à un marché du travail tendu ? La littérature économique ne permet que partiellement de répondre à cette question. Dans le modèle d'appariement canonique des lauréats du prix Nobel d'économie 2010 pour leurs travaux sur le chômage, Peter Diamond, Dale Mortensen et Christopher Pissarides, aucun lien n'existe entre tension sur le marché du travail et effort de recherche des employeurs, ce dernier ne variant pas au cours du cycle économique. Néanmoins, Steven J. DAVIS et ses co-auteurs (2013) ont mis en évidence que la baisse persistante de l'intensité agrégée de recrutement des employeurs ${ }^{6}$ pouvait être à l'origine de la relative atonie du taux d'embauche aux États-Unis après la crise de 2008, malgré la hausse de la part des emplois vacants. Selon Alessandro GAVAZZA et ses co-auteurs (2018), ce lien entre intensité (effort) de recrutement et cycle économique transite, en partie, par la tension sur le marché du travail. En effet, à la suite d'un choc négatif, la tension diminue - car le nombre de personnes en recherche d'emploi par poste vacant devient plus élevé - et les employeurs ont moins de difficultés pour pourvoir leurs postes vacants. Ils consacrent dès lors moins de dépenses au recrutement, ce qui induit une baisse de l'intensité agrégée de recrutement. Les employeurs, par les méthodes qu'ils mettent en œuvre pour attirer des candidats, sont donc plus ou moins en mesure d'accélérer ou de ralentir le processus d'appariement entre offre et demande d'emploi sur le marché du travail. Même si la littérature sur l'employer search (recherche de candidats par

6. Cette intensité (effort) correspond à l'agrégation de l'ensemble des instruments utilisés par les différents employeurs pour recruter: le niveau de salaire offert, l'éventuel ajustement de leurs critères de recrutement, le nombre de canaux activés, les méthodes utilisées pour sélectionner les candidats, etc. On parlera indifféremment d'effort/intensité du recrutement. 
l'employeur) est beaucoup moins développée que celle sur le job search (recherche d'emploi par les demandeurs d'emploi ou les personnes déjà en emploi), certains travaux avaient déjà observé que les employeurs choisissaient des canaux de recherche de candidats - relations, annonces, intermédiaires du marché du travail, etc. - différents selon le contexte du marché du travail sur lequel ils recrutent, et notamment son degré de tension (Russo et al., 2000; Bessy, MARChal, 2007; Estrade, 2013; BERGEAT, RÉMY, 2019).

Enfin, que sait-on des relations entre efforts de recherche des employeurs et difficultés de recrutement, le troisième et dernier volet de notre triptyque? Si des études analysent les liens entre effort des recruteurs - mesuré seulement par les canaux activés - et durée du recrutement, elles ne s'intéressent pas spécifiquement aux liens entre effort et difficultés. Pourtant, il y a bien un lien entre durée et difficulté puisqu'un allongement du temps nécessaire pour pourvoir un poste vacant peut être le signe de difficultés d'embauche. Les recrutements effectués par annonces sont les plus longs (RoPer, 1988; Gorter et al., 1996; Russo et al., 2000; DeVAro, 2005) tandis que ceux réalisés grâce aux candidatures spontanées (Russo et al., 2000; DEVARO, 2005) ou aux relations (ROPER, 1988; GORTER et al., 1996) sont les plus rapides. De plus, le recours à davantage de canaux va de pair avec un allongement du processus de recrutement (Russo et al., 2000). Il existe ainsi bien une relation entre durée du recrutement et effort des employeurs, mesuré par le nombre de canaux utilisés. Néanmoins, les liens entre la durée (ou la difficulté) de l'embauche et les autres étapes du recrutement (méthodes utilisées pour sélectionner les candidats, concessions que sont prêts à réaliser les recruteurs, etc.) n'ont pas fait l'objet de travaux à notre connaissance.

À partir de l'enquête Offre d'emploi et recrutement (Ofer) 2016 (encadré 1), cette étude vise à répondre aux questions suivantes : recruter sur un marché du travail tendu est-il systématiquement synonyme de difficultés de recrutement ? La nature des difficultés rencontrées (inadéquation entre les profils des candidats et les exigences du poste, manque de candidatures, déficit d'image du métier, etc.) diffère-t-elle selon le contexte du segment du marché du travail sur lequel l'employeur embauche? Les comportements des employeurs - notamment la manière dont ils formalisent leurs besoins, recherchent et sélectionnent les candidats - varient-ils selon ce contexte et les difficultés auxquelles ils font face? Comment justement définir et tenir compte du «contexte » dans lequel s'inscrivent les recrutements?

L'enquête Ofer 2016 - menée par le ministère en charge du travail auprès d'employeurs ayant recruté entre septembre et novembre 2015 en contrat à durée indéterminée (CDI) ou en contrat à durée déterminée (CDD) de plus d'un mois - est particulièrement appropriée pour aborder ces questions car elle permet non seulement de quantifier leurs difficultés pour embaucher, mais également de mettre ces difficultés en regard des moyens déployés au cours du processus de recrutement. En effet, des questions détaillées sont posées sur les sources de ces difficultés, l'ensemble des actions mises en œuvre au cours de l'embauche (canaux de recherche de candidats mobilisés, méthodes de sélection utilisées et intensité de cette sélection, nombre de personnes 
impliquées, etc.) ainsi que sur leurs résultats (nombre de candidatures examinées, coût et durée du recrutement, etc.). Des informations sont également disponibles sur les établissements recruteurs (taille, secteur, ancienneté, etc.) ainsi que sur leurs postes à pourvoir (qualification, contrat de travail, métier, etc.).

Pour déterminer dans quelle mesure le contexte du marché du travail peut influer sur les difficultés effectivement rencontrées par les employeurs et sur leurs efforts de recrutement, nous adoptons une démarche originale synthétisée dans le schéma ci-dessous. Nous avons défini ce que nous appelons un contexte global de tension à partir de quatre indicateurs mesurés au niveau du métier: les trois premiers - la tension, le nombre d'emplois vacants par salarié et le taux de sortie du chômage (ici calculé à partir des sorties de liste des demandeurs d'emploi) - sont issus d'une source administrative, la Statistique du marché du travail de Pôle Emploi et de la Dares. Le quatrième indicateur - la part des projets de recrutement anticipés comme difficiles par les employeurs - est issu de l'enquête Besoins en main-d' æuvre de Pôle emploi (encadré 1). Afin de résumer ce contexte global de tension prévalant sur les différents segments de marché du travail (chaque segment correspondant à un métier donné), nous avons construit une typologie des métiers à l'aide de ces quatre indicateurs. Pour identifier l'effet propre du contexte global de tension, nous mettons ensuite cette typologie des métiers en regard des difficultés de recrutement éventuellement rencontrées ex post par les employeurs et des moyens qu'ils mobilisent pour mener à bien le recrutement, difficultés et moyens étant mesurés à partir de l'enquête Ofer 2016. Cette enquête permet, en effet, de décomposer l'effort de recrutement des employeurs et de lier chacune de leurs actions en la matière aux différents contextes globaux

\section{SCHÉMA - Les différentes étapes de l'étude}

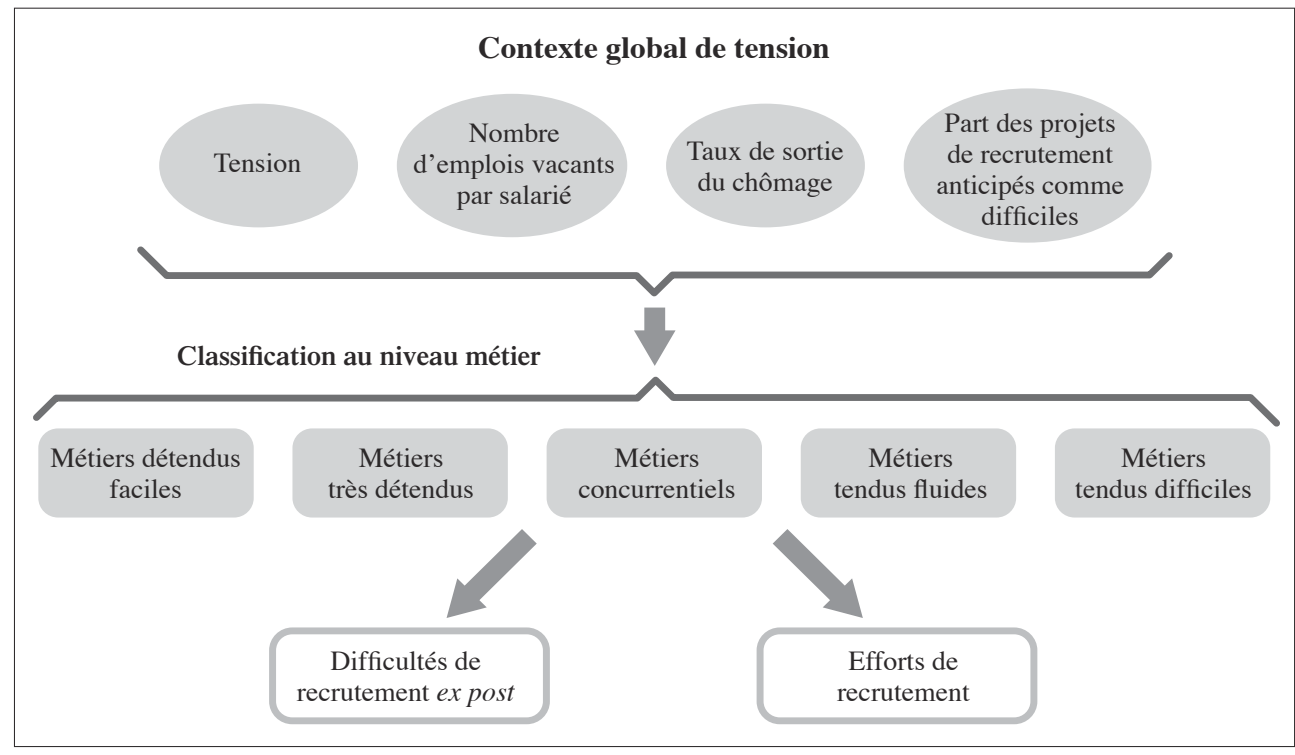


de tension synthétisés par notre typologie de métiers, à la différence des travaux de S. J. DAVIS et ses co-auteurs (2013) qui étudient cet effort de manière globale. Mais ce n'est pas le seul atout de cette enquête grâce à laquelle il est possible d'améliorer la qualité de la mesure des tensions. L'indicateur utilisé traditionnellement rapporte le flux d'offres d'emploi intermédiées par Pôle emploi (une mesure du nombre d'emplois vacants) au flux de demandeurs d'emploi inscrits à Pôle emploi. L'une des limites de cet indicateur est qu'il ne porte que sur les offres d'emploi déposées à Pôle emploi et non sur l'ensemble des postes à pourvoir. L'enquête Ofer permet d'estimer au niveau de chaque métier la part des recrutements impliquant la mobilisation de Pôle emploi et de s'approcher ainsi d'un indicateur du nombre total d'emplois vacants.

\section{ENCADRÉ 1 \\ L'enquête Offre d'emploi et recrutement (Ofer) 2016 et les autres sources mobilisées}

L'enquête Offre d'emploi et recrutement (Ofer) a été menée par la Dares en 2016 auprès d'établissements d'au moins un salarié du secteur concurrentiel non agricole ayant nouvellement recruté ${ }^{1}$ en CDI ou en CDD de plus d'un mois entre septembre et novembre 2015. Réalisée à la demande de nombreux partenaires de la Dares, cette enquête vise à renouveler l'analyse du processus de recrutement et à améliorer la compréhension des mécanismes d'appariement sur le marché du travail depuis la dernière enquête de 2005. Ofer 2016 est la seule enquête au niveau national à décrire précisément les différentes étapes du recrutement, de la formulation du besoin en ressources humaines jusqu'à la satisfaction ou non de l'employeur à l'égard du recrutement réalisé. Contrairement à la précédente enquête (en 2005), elle n'étudie que les embauches effectivement réalisées et n'analyse pas les recrutements n'ayant pas abouti.

Pour réduire les biais de mémoire, l'interrogation a porté sur un recrutement précis, relativement peu de temps après ce dernier (quatre à dix mois) et sur des contrats d'au moins un mois. Le taux de réponse à l'enquête est de $64 \%$, soit 8510 répondants. Le champ de notre étude a été restreint aux établissements de France métropolitaine recrutant sur des postes n'appartenant pas aux domaines professionnels suivants: A (agriculture, marine, pêche), $\mathrm{P}$ (administration publique, professions juridiques, armée et police) et $\mathrm{X}$ (politique, religion); en effet, ces domaines professionnels sont moins bien couverts que les autres par les données complémentaires que nous mobilisons. Au total, l'échantillon retenu dans notre article comprend 8063 établissements répondants représentant environ 1126500 recrutements. L'enquête Ofer 2016 comporte, entre autres, des informations détaillées sur les difficultés de recrutement rencontrées ex post par les employeurs ainsi que sur leurs efforts de recrutement (canaux de recherche de candidats mobilisés, méthodes de sélection utilisées, pièces demandées aux candidats, etc.). De nombreuses variables

1. Pour être retenue dans le champ de l'enquête $O f e r$, une personne ayant déjà été salariée de l'établissement enquêté doit remplir deux critères : sa précédente embauche doit dater d'au moins deux ans par rapport à la date de l'enquête et elle doit avoir quitté l'établissement depuis au moins six mois. Pour une présentation détaillée de l'enquête, voir l'encadré 1 de BERGEAT, RÉMY (2017). 
sont également disponibles sur le poste à pourvoir (qualification, temps de travail, etc.) et l'établissement (taille, secteur, etc.). Cette enquête ne permet pas de mener une analyse au niveau local (bassin d'emploi notamment), même s'il aurait été intéressant de pouvoir croiser ce niveau avec la dimension métier pour mieux rendre compte de la réalité des différents marchés du travail.

Pour calculer les indicateurs de contexte du marché du travail au niveau du métier, nous avons utilisé deux sources principales:

1. La Statistique du marché du travail (STMT) est une source exhaustive issue des fichiers de gestion de Pôle emploi, produite par la Dares et Pôle emploi. Cette source permet de recenser les effectifs de demandeurs d'emploi présents en fin de mois, selon leurs caractéristiques (catégorie et ancienneté d'inscription sur les listes, âge, etc.) ainsi que les offres d'emploi passant par Pôle emploi. Elle retrace également les flux d'entrées et de sorties des demandeurs d'emploi inscrits à Pôle emploi ainsi que les enregistrements et les sorties des offres d'emploi. Cette source permet de calculer trois de nos indicateurs : la tension mesurée en stock (nombre d'emplois vacants/nombre de demandeurs d'emploi sur un métier donné), le nombre d'emplois vacants par salarié et le taux de sortie du chômage.

2. L'enquête Besoins en main-d'œuvre (BMO) de Pôle emploi est menée chaque année auprès d'environ 1,6 million d'établissements du secteur privé (y compris agriculture) et d'une partie du secteur public (collectivités territoriales, établissements publics administratifs et offices publics ou ministériels) sur l'ensemble de la France. Avec un taux de réponse de $25 \%$, l'échantillon compte 400000 établissements répondants. L'enquête $B M O$ recense les intentions de recrutement des employeurs pour l'année à venir, qu'il s'agisse de créations de poste ou de remplacements, ainsi que leurs difficultés anticipées de recrutement. Notre étude mobilise précisément l'enquête $B M O 2015$ réalisée entre octobre et décembre 2014 auprès de 402500 établissements afin de calculer la part des projets de recrutements anticipés comme difficiles.

Enfin, nous avons mobilisé l'enquête Emploi de l'Insee - plus précisément les millésimes 2014 à 2016 - pour calculer le niveau d'emploi salarié moyen associé à un métier donné. Cette enquête est réalisée chaque trimestre par l'Insee auprès d'environ 110000 personnes de 15 ans ou plus sur l'ensemble de la France. Sa présentation détaillée est disponible sur le site internet de l'Insee : https://www.insee.fr/fr/metadonnees/source/ serie/s1223, consulté le 7 octobre 2021.

Nous présentons dans une première partie les quatre indicateurs retenus pour caractériser le contexte global de tension associé à un métier donné, et nous décrivons la typologie des cinq classes de métiers. La construction de cette typologie permet de synthétiser les différents contextes globaux de tension auxquels sont confrontés les employeurs lorsqu'ils recrutent sur tel ou tel type de métier. En appliquant cette typologie aux données de l'enquête $O f e r$, il est possible de caractériser les recrutements - selon le type de poste et d'établissement - de chaque classe de métiers. Les liens entre contexte global de tension et difficultés de recrutement déclarées ex post par les employeurs font l'objet d'une deuxième partie, qui montre qu' un marché du travail tendu n'induit pas nécessairement des difficultés de recrutement. Ainsi, parmi les employeurs 
des deux classes de métiers les plus tendus, seuls ceux de la classe des «métiers tendus difficiles », comportant la tension et les difficultés d'embauche anticipées les plus élevées, déclarent ex post en avoir rencontré davantage. Enfin, la troisième partie permet d'analyser les liens entre contexte global de tension et actions mises en œuvre par les employeurs pour recruter (canaux de recherche activés, méthodes de sélection utilisées, organisation du recrutement, concessions réalisées, etc.). Nous mettons là aussi en évidence le fait qu'il n'y a pas de liens univoques. Ainsi, les recruteurs des deux classes de métiers les plus tendus ont demandé davantage de pièces aux candidats et en ont reçu une plus grande part en entretien individuel que ceux de la classe des «métiers détendus faciles ». A contrario, ils n'ont pas mobilisé davantage de canaux de recherche de candidats ou de méthodes de sélection que ceux des autres classes.

\section{Indicateurs retenus pour mesurer le contexte global de tension et typologie des métiers}

Notre étude vise à analyser dans quelle mesure les recruteurs intervenant sur un marché du travail plus ou moins tendu traduisent cette situation dans leur déclaration de difficultés de recrutement et adaptent leur comportement d'embauche en conséquence. Autrement dit, nous nous interrogeons sur la nature des difficultés de recrutement déclarées par les employeurs au regard de la situation prévalant sur le marché du travail sur lequel ils recrutent. Nous avons choisi de mener une analyse au niveau métier et plus précisément, au niveau des familles professionnelles ${ }^{7}$ (Fap 87). En effet, ce niveau nous semble particulièrement pertinent pour juger de l'adéquation entre l'offre et la demande d'emploi ${ }^{8}$ et d'éventuelles pénuries de candidats ou de postes. Des travaux américains récents (ŞAHIN et al., 2014) ont ainsi mis en évidence que l'inadéquation entre offre et demande d'emploi au niveau du métier expliquait $29 \%$ de la hausse du chômage entre début 2006 et fin 2009. Sur une plus longue période et toujours aux États-Unis (1979-2015), Benedikt HerZ et Thijs van RENS (2018) ont montré que cette inadéquation était responsable d'un cinquième des fluctuations du chômage.

7. Cette nomenclature a été conçue par la Direction de l'animation de la recherche, des études et des statistiques (Dares) pour avoir une approche unifiée des « métiers » dans l'ensemble des statistiques de l'emploi, en niveau et en flux. Elle constitue une table de passage entre la profession et catégorie socioprofessionnelle (PCS) utilisée par l'Insee pour coder la profession dans les sources statistiques de l'emploi et le Répertoire opérationnel des métiers et des emplois (ROME) mobilisé par Pôle emploi pour coder le métier dans une logique opérationnelle de rapprochement entre emplois recherchés par les demandeurs d'emploi et offres déposées par les entreprises. La version utilisée dans cette étude (Fap-2009) fait ainsi le lien entre la PCS-2003 de l'Insee et le ROME-2009. Pour une présentation détaillée, voir le site de la Dares : https://dares.travail-emploi.gouv.fr/donnees/la-nomenclature-des-familles-professionnelles-fap-2009. Ces familles professionnelles détaillées sont regroupées en 87 postes (Fap 87).

8. Cela signifie que les compétences des personnes en recherche d'emploi - en matière de diplôme, formation, expérience, compétences techniques ou encore de qualités personnelles - correspondent aux critères de recrutement des employeurs sur les postes qu'ils cherchent à pourvoir. 


\section{Les indicateurs permettant de définir un contexte global de tension sur le marché du travail}

Pour mesurer la tension prévalant sur un segment du marché du travail donné (correspondant ici à un métier donné), nous avons retenu plusieurs indicateurs (graphique 1), afin de synthétiser le contexte du segment de marché sur lequel l'employeur est amené à recruter. Nous regroupons les métiers dont les indicateurs sont proches et menons notre analyse dans un cadre simplifié. Les différents segments de marchés du travail définis par les métiers ont, par ailleurs, des modes de fonctionnement très hétérogènes, qu'il s'agisse du lien formation-emploi, de la mobilité professionnelle, des conditions de travail et d'emploi associées, etc., mais nous nous limitons dans cette étude à les comparer au regard de leur seul contexte global de tension.

Le premier indicateur retenu est logiquement la mesure habituelle de la tension sur le marché du travail, correspondant au ratio entre le nombre d'emplois vacants proposés par les employeurs et le nombre de personnes en recherche d'emploi pour un métier donné: c'est la tension mesurée en stock. Plus cet indicateur est élevé, plus la concurrence entre employeurs est intense et plus ces derniers rencontreront des difficultés pour pourvoir leur poste vacant rapidement (et plus les personnes en recherche d'emploi trouveront un poste facilement). A contrario, plus cet indicateur est faible, traduisant une pénurie de postes à pourvoir et/ou une abondance de personnes

GRAPHIQUE 1 - Les indicateurs construits pour rendre compte du contexte global de tension

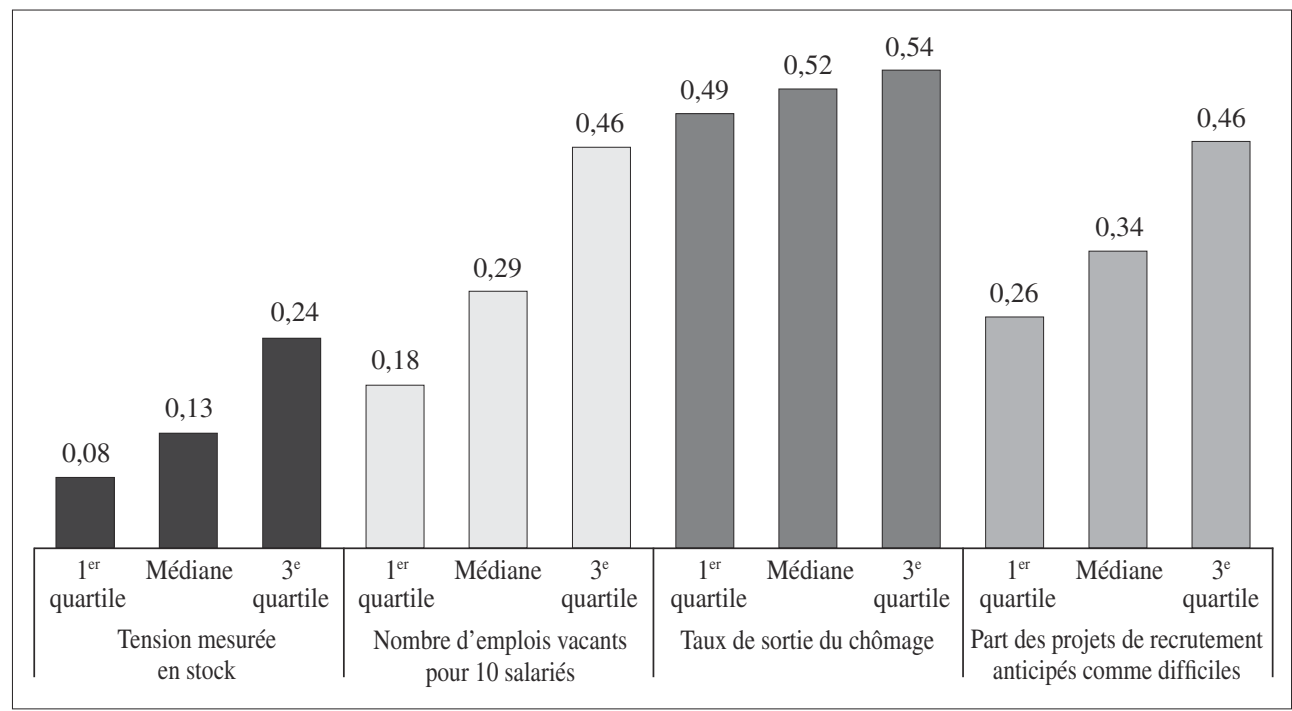

Note : les métiers sont pondérés par les effectifs salariés.

Lecture : la tension mesurée en stock est inférieure ou égale à 0,08 pour $25 \%$ des métiers, c'est-à-dire pour le $1^{\mathrm{er}}$ quartile.

Champ : emploi salarié, hors domaines professionnels A (agriculture, marine, pêche), P (administration publique, professions juridiques, armée et police) et X (politique, religion); France métropolitaine.

Sources: Insee, enquêtes Emploi 2014, 2015 et 2016; Pôle emploi et Dares, STMT; Pôle emploi, enquête Besoins en main-d'œuvre et Dares, enquête Ofer 2016. 
en recherche d'emploi et donc de candidats, plus les recruteurs pourvoiront leur poste rapidement et plus les personnes en recherche d'emploi devront attendre avant de trouver un emploi.

Nous cherchons à aller au-delà de la mesure habituelle de cet indicateur de tension. Ainsi, pour calculer le numérateur (le nombre d'emplois vacants) - qui est également le numérateur de notre deuxième indicateur correspondant au nombre d'emplois vacants par salarié -, nous disposons de deux sources potentielles: les offres collectées par Pôle emploi et non satisfaites, mesurées à partir de la Statistique du marché du travail de Pôle emploi et de la Dares (STMT, encadré 1) et les emplois vacants de l'enquête Activité et conditions d'emploi de la main-d'œuvre (Acemo) de la Dares. Nous avons retenu la première source car le nombre d'emplois vacants estimé à partir de l'enquête Acemo n'est pas déclinable par métier et est susceptible d'être sous-estimé (FonDEUR, ZANDA, 2009; COE, 2013), les répondants pouvant mal interpréter la question posée sur leurs emplois à pourvoir. Le nombre d'offres collectées par Pôle emploi présente l'avantage de résulter d'un acte de dépôt d'une offre par les entreprises plutôt que de déclarations, mais n'est pas non plus exempt de critiques (CoE, 2013). Il est certes mesurable au niveau du métier mais peut conduire à surestimer le nombre d'emplois à pourvoir dans la mesure où certaines offres pourvues ou abandonnées peuvent ne pas avoir été signalées par le recruteur. Cette limite est néanmoins beaucoup moins prégnante que par le passé puisque Pôle emploi a mis en place depuis 2013 une procédure de clôture automatique des offres en l'absence de réponse de l'employeur. Surtout, ces offres ne tiennent compte que des emplois vacants qui font l'objet d'une diffusion auprès de Pôle emploi. Pour surmonter cette difficulté, nous avons divisé le nombre d'offres collectées par Pôle emploi et non satisfaites en fin de trimestre par la part des recrutements intermédiés par Pôle emploi, calculée à partir d'Ofer $2016^{9}$ (39\% en moyenne ${ }^{10}$ ), et ainsi obtenu un nombre total d'emplois vacants indépendant du canal utilisé par l'employeur pour rechercher des candidats. Lorsque les effectifs de l'enquête le permettent, cette part est déclinée au niveau Fap 87; lorsque les métiers sont plus rares, elle l'est à un niveau plus agrégé (au niveau Fap en 22 postes).

Pour calculer le dénominateur de la tension, nous avons utilisé le nombre de demandeurs d'emploi inscrits à Pôle emploi en catégorie $\mathrm{A}, \mathrm{B}, \mathrm{C}^{11}$ à la fin du trimestre calculé à partir de la STMT. Cette mesure n'inclut pas les recherches d'emploi des personnes en poste et non inscrites à Pôle emploi. Finalement, l'indicateur de tension retenu

\footnotetext{
9. Une limite potentielle de cette méthode est que l'on applique la part de Pôle emploi dans les recrutements (emplois vacants pourvus) à un stock d'emplois vacants. Néanmoins la part de Pôle emploi dans ce stock étant à notre connaissance indisponible, il n'est pas possible de faire mieux.

10. Pour 7 familles professionnelles sur les 59 analysées, dont les ingénieurs en informatique, les techniciens et agents de maîtrise des industries de process et les professionnels de la communication et de l'information, cette part ne dépasse pas $20 \%$. À l'opposé, pour 6 familles professionnelles, dont les secrétaires de direction, les agents de gardiennage et de sécurité et les employés de maison, cette part est supérieure ou égale à $60 \%$.

11. Ces catégories correspondent respectivement aux demandeurs d'emploi tenus de faire des actes positifs de recherche d'emploi et sans emploi au cours du mois (A), ayant exercé une activité réduite courte de 78 heures ou moins (B), et ayant exercé une activité réduite longue de plus de 78 heures (C).
} 
dans cette étude (graphique 1) se distingue de celui diffusé par la Dares jusqu'en 2017 (BERGEAT, 2017). Au numérateur et au dénominateur, il porte sur des niveaux en fin de trimestre et non sur des flux, les données utilisées sont annualisées et non trimestrielles. L'indicateur en stock est moins volatil que celui en flux (même si les stocks dépendent bien sûr des flux). Enfin, le champ des emplois vacants considéré est plus large que celui des seules offres collectées par Pôle emploi. Le fait de diviser, pour calculer le numérateur de notre indicateur, le nombre d'offres collectées par Pôle emploi et non satisfaites en fin de trimestre par la part de Pôle emploi dans les embauches (mesurée à partir d'Ofer) permet d'éviter la critique usuellement adressée à l'indicateur de tension, à qui il est reproché d'être davantage une mesure de la part de marché de Pôle emploi sur un segment donné du marché du travail que de la tension effective sur ce segment.

Néanmoins, cet indicateur de tension ne suffit pas à rendre compte de l'ensemble de la situation sur le marché du travail et ne permet notamment pas de savoir si un niveau de tension élevé est le fait d'une pénurie de personnes en recherche d'emploi ou d'un nombre trop important de postes à pourvoir. Nous retenons ainsi un deuxième indicateur: le nombre d'emplois vacants par salarié correspondant plus précisément au nombre d'emplois vacants pour dix salariés. Le numérateur est le même que celui de la tension mesurée en stock, et le dénominateur est le niveau d'emploi salarié moyen associé à un métier donné, calculé à partir de la moyenne des années 2014 à 2016 de l'enquête Emploi de l'Insee pour améliorer la précision des résultats. Si une forte tension (décrite par le premier indicateur) s'accompagne d'un nombre d'emplois vacants par salarié élevé par rapport à la moyenne (deuxième indicateur), cela signifie qu'elle tiendrait plutôt à une concurrence entre recruteurs qu'à une pénurie de candidats. Cette part élevée d'emplois vacants peut résulter d'une croissance de l'activité des entreprises recrutant sur ce métier, mais aussi d'un turn-over important sur des emplois courts ou de mécanismes de fuite des salariés en raison de conditions de travail difficiles.

En complément, nous utilisons un troisième indicateur évalué à partir de la STMT: le taux de sortie du chômage qui permet de rendre compte de la fluidité du marché du travail du point de vue des demandeurs d'emploi. Un taux élevé signifie qu'une forte part des demandeurs d'emploi inscrits est sortie des listes de Pôle emploi au cours de l'année écoulée. Il ne peut être strictement assimilé à un taux de sortie vers l'emploi, car, en 2015, seules $42 \%$ des sorties de liste correspondent à la reprise d'un emploi d'après l'enquête Sortants de la Dares et de Pôle emploi ${ }^{12}$ (BAgein, Poujouly, 2017), mais il en donne néanmoins une indication. Plus précisément, ce taux est calculé comme le rapport entre les sorties de liste des demandeurs d'emploi des catégories A, B, C au cours des 12 derniers mois et le nombre de demandeurs d'emploi susceptibles d'en sortir au cours de cette période (c'est-à-dire déjà présents 12 mois auparavant ou entrés au cours des 12 mois considérés).

12. Plus précisément, d'après l'enquête de 2015, $42 \%$ des sortants ont trouvé un emploi, $13 \%$ ont commencé une formation, $18 \%$ sont sortis pour un défaut d'actualisation suivi d'une réinscription dans les trois mois à Pôle emploi et les $27 \%$ restants ont arrêté définitivement ou temporairement leurs recherches d'emploi via Pôle emploi, ce qui peut correspondre selon les cas à une sortie ou non de la population active. 
Ces trois premiers indicateurs - tension proprement dite, nombre d'emplois vacants par salarié et taux de sortie du chômage - sont annualisés sur la base des quatre trimestres de 2015 afin d'en limiter la volatilité saisonnière. Pour compléter notre analyse, nous avons eu recours à un dernier indicateur plus subjectif que les autres: la part des recrutements anticipés comme difficiles par les recruteurs, estimée à partir de l'enquête Besoins en main-d'œuvre de Pôle emploi de 2015 (encadré 1). En effet, un marché du travail tendu n'est pas nécessairement synonyme de difficultés de recrutement si l'offre de travail est en adéquation avec la demande de travail et/ ou est en mesure de s'ajuster rapidement. Il nous semblait dès lors intéressant de compléter nos indicateurs objectifs de la tension par la perception des employeurs à l'égard du segment de marché sur lequel ils sont amenés à recruter: cette perception est en effet susceptible d'influer sur leur comportement et les moyens qu'ils mettent en œuvre lors de l'embauche. S'ils anticipent un recrutement difficile, ils vont ainsi peut-être envisager d'y consacrer davantage de temps, de solliciter plus activement leurs canaux de recherche de candidats habituels, etc. Les difficultés qu'anticipent les recruteurs sont souvent surestimées par rapport aux difficultés qu'ils rencontrent effectivement, comme le montre l'enquête complémentaire de Pôle emploi, un écart pouvant en partie s'expliquer par le fait que les recruteurs sous-estiment fortement les recrutements qu'ils auront à réaliser (remplacements non anticipables, contrats courts non prévus, etc.) (COE, 2013).

Ces quatre indicateurs nous permettent de définir le contexte du marché du travail associé à chaque métier et de construire notre typologie de métiers en regroupant les métiers dont les contextes sont les plus proches (voir partie suivante). Nous mettons ensuite en regard ces indicateurs de situation sur le marché du travail avec des indicateurs de difficultés de recrutement mentionnées ex post par les recruteurs et des indicateurs de leurs efforts d'embauche, tous construits à partir de l'enquête Ofer 2016.

\section{Cinq classes de métiers au regard du contexte global de tension}

Afin de pouvoir analyser les différents contextes de marché du travail auxquels les employeurs sont confrontés selon le type de métier sur lequel ils recrutent, une typologie a été élaborée à l'aide d'une classification ascendante hiérarchique (encadré 2). Cette typologie permet de regrouper les métiers ayant les contextes les plus proches au vu des indicateurs construits (voir point précédent et graphique 1) et de limiter l'hétérogénéité de l'information à analyser. Cinq classes de métiers sont ainsi identifiées selon ces indicateurs.

La classe des «métiers détendus faciles » est marquée par les difficultés de recrutement anticipées par les employeurs les plus basses, un indicateur de tension mesurée en stock ${ }^{13}$ faible et une concurrence entre recruteurs sur les emplois vacants

13. Dans la suite du texte, nous nommerons plus simplement l'indicateur de tension mesurée en stock « indicateur de tension». 


\section{ENCADRÉ 2}

\section{La construction de la typologie des métiers}

La typologie a été construite en deux étapes. Une analyse des correspondances multiples (ACM) a d'abord permis, à partir des relations entre les différentes variables retenues pour l'analyse, de projeter les données relatives au contexte global de tension des métiers - familles professionnelles (Fap) - dans un espace de dimension plus réduite, structuré autour de différents axes. Les quatre premiers axes de l'ACM, qui résument $53 \%$ de l'information initiale, ont été retenus. Les variables actives de l'ACM sont les quatre indicateurs choisis pour rendre compte de la tension sur le marché du travail:

- la tension mesurée en stock;

- le nombre d'emplois vacants par salarié (plus précisément pour dix salariés);

- le taux de sortie du chômage des demandeurs d'emploi ;

- la part des projets de recrutement anticipés comme difficiles.

Les trois premiers indicateurs sont estimés à partir de la Statistique du marché $d u$ travail de Pôle emploi et de la Dares (STMT, encadré 1) tandis que le dernier est calculé à partir de l'enquête Besoins en main-d'œuvre de Pôle emploi.

Afin de limiter l'influence de valeurs extrêmes, ces quatre indicateurs continus, déclinés au niveau Fap 87 et mesurés sur l'année 2015, ont été transformés en variables ordinales comptant soit des terciles de tranches (le taux de sortie du chômage, la part des projets de recrutement anticipés comme difficiles), soit des quintiles (la tension, le nombre d'emplois vacants par salarié), selon leur dispersion initiale (graphique 1).

Dans un second temps, les coordonnées des métiers sur les quatre axes retenus ont été utilisées pour réaliser la classification ascendante hiérarchique $(\mathrm{CAH})$ des métiers. La méthode retenue pour regrouper les individus est la méthode de Ward qui vise à minimiser l'inertie au sein des classes et à maximiser l'inertie entre les classes afin d'obtenir les classes les plus homogènes possibles en pondérant les métiers par leur poids dans l'emploi total. La classification des métiers est construite à partir d'indicateurs mesurés sur le marché du travail dans son ensemble, ce qui permet de ne pas restreindre l'analyse au champ spécifique d'Ofer 2016.

Des variables supplémentaires, non mobilisées lors de la construction des classes et détaillées dans le tableau $1 \mathrm{~A}$ en annexe, ont ensuite été introduites pour mieux décrire les différentes classes de métiers : les caractéristiques des recrutements réalisés (temps de travail associé, catégorie socioprofessionnelle du poste, type de contrat de travail, etc.) et des établissements à l'origine de ces recrutements (taille, secteur d'activité, ancienneté, etc.). Pour disposer de ces variables supplémentaires, nous avons apparié les classes de métiers avec les recrutements de l'enquête Ofer 2016 classés par Fap.

- mesurée par le nombre d'emplois vacants pour dix salariés - relativement limitée (tableau 1). Elle représente la plus forte part de l'emploi salarié et des recrutements, soit respectivement $31 \%$ et $38 \%$ d'entre eux.

Cette classe compte majoritairement des postes d'employés, qualifiés - employés administratifs d'entreprises, secrétaires, aides-soignants - ou non - vendeurs, caissiers, 
employés de libre-service (graphique 2, p. 61). Les conducteurs de véhicules, les ouvriers qualifiés de la maintenance et non qualifiés du gros œuvre et du second œuvre du bâtiment et des travaux publics représentent près du tiers des recrutements de la classe.

Les établissements qui recrutent sur un poste correspondant à un « métier détendu facile » sont plus souvent positionnés sur les secteurs du commerce et du transport et, dans une moindre mesure, sur celui de la construction (tableau 1A en annexe). Ils appartiennent plus souvent à un groupe ou à une entreprise multi-établissements et sont les moins nombreux à faire appel aux contrats très courts ${ }^{14}$, même s'ils recrutent un peu plus souvent en CDD d'un à trois mois que les autres.

La classe des « métiers très détendus » se caractérise par l'indicateur de tension le plus faible $(0,07)$. Le faible niveau de cet indicateur de tension est le résultat de la concurrence sur les postes vacants la plus limitée (au numérateur de cet indicateur) et du nombre de demandeurs d'emploi par salarié (plus précisément pour dix salariés dans le tableau 1) le plus conséquent (au dénominateur). Les employeurs peuvent ainsi trouver facilement des candidats sur le métier considéré pour autant que ces derniers aient les compétences demandées et résident sur le même territoire. Le taux de sortie du chômage est d'ailleurs le plus bas dans cette classe, les emplois proposés n'étant pas suffisamment nombreux pour que les demandeurs d'emploi puissent sortir rapidement du chômage. Malgré cette abondance de candidats potentiels, les difficultés de recrutement anticipées par les employeurs sont dans la moyenne. Cette classe, représentant $23 \%$ de l'emploi salarié et $18 \%$ des recrutements, est celle où Pôle emploi intervient le plus souvent dans les embauches.

Les postes sont plutôt non qualifiés, soit des postes d'employés - aides à domicile et aides ménagères, agents de gardiennage et de sécurité, assistantes maternelles -, soit d'ouvriers - dans les industries de process et la manutention, agents d'entretien. Cette classe compte aussi les postes d'ouvriers qualifiés de la manutention et de techniciens des services administratifs, comptables et financiers. Les conditions de travail semblent globalement plus difficiles que celles des autres $\operatorname{classes}^{15}:$ les personnes exerçant les métiers de cette classe déclarent plus souvent avoir au moins trois contraintes physiques et exécuter des tâches répétitives. Elles sont moins nombreuses à émettre le souhait de transmettre leur métier à leurs enfants.

Les établissements qui recrutent sur les métiers de cette classe appartiennent plus souvent aux secteurs des services aux entreprises et des services non marchands et comptent plus souvent dix salariés ou plus. Plus rarement localisés dans l'aire urbaine de Paris, ces établissements recrutent plus souvent simultanément sur plusieurs postes de même catégorie, à temps partiel et dans une moindre mesure, en contrat d'un à

14. L'enquête $O f e r 2016$ ne porte que sur les recrutements en CDD de plus d'un mois mais les recruteurs sont interrogés sur leur usage global des contrats de moins d'un mois au cours de l'année 2015.

15. Ces conditions de travail sont mesurées au niveau de la Fap à partir de l'enquête Conditions de travail - risques psychosociaux 2016 réalisée par la Dares, la Direction de la recherche, des études, de l'évaluation et des statistiques (Drees) et la Direction générale de l'administration et de la fonction publique (DGAFP), voir tableau 1B en annexe. 
TABLEAU 1 - Les cinq classes de métiers selon les indicateurs de contexte global de tension

\begin{tabular}{|c|c|c|c|c|c|c|}
\hline & $\begin{array}{l}\text { Métiers } \\
\text { détendus } \\
\text { faciles }\end{array}$ & $\begin{array}{l}\text { Métiers } \\
\text { très } \\
\text { détendus }\end{array}$ & $\begin{array}{l}\text { Métiers } \\
\text { concur- } \\
\text { rentiels }\end{array}$ & $\begin{array}{l}\text { Métiers } \\
\text { tendus } \\
\text { fluides }\end{array}$ & $\begin{array}{c}\text { Métiers } \\
\text { tendus } \\
\text { difficiles }\end{array}$ & Ensemble \\
\hline Tension mesurée en stock (1)/(2) & 0,13 & 0,07 & 0,17 & 0,22 & 0,34 & 0,17 \\
\hline $\begin{array}{l}\text { Nombre d'emplois vacants } \\
\text { pour dix salariés (1) }\end{array}$ & 0,30 & 0,19 & 0,54 & 0,44 & 0,43 & 0,36 \\
\hline Taux de sortie du chômage & 0,52 & 0,48 & 0,51 & 0,57 & 0,52 & 0,52 \\
\hline $\begin{array}{l}\text { Part des projets de recrutement anticipés } \\
\text { comme difficiles }\end{array}$ & 0,28 & 0,35 & 0,35 & 0,41 & 0,51 & 0,36 \\
\hline $\begin{array}{l}\text { Nombre de demandeurs d'emploi } \\
\text { pour dix salariés (2) }\end{array}$ & 2,88 & 3,22 & 3,02 & 2,05 & 1,51 & 2,68 \\
\hline
\end{tabular}

Note : les métiers sont pondérés par les effectifs salariés.

Lecture: dans la classe des «métiers détendus faciles », seuls $28 \%$ des projets de recrutement sont anticipés comme difficiles par les employeurs contre $36 \%$ pour l'ensemble.

Champ : emploi salarié, hors domaines professionnels A (agriculture, marine, pêche), $\mathrm{P}$ (administration publique, professions juridiques, armée et police) et X (politique, religion); France métropolitaine.

Sources: Insee, enquêtes Emploi 2014, 2015 et 2016; Pôle emploi et Dares, STMT; Pôle emploi, enquête Besoins en main-d'œuvre.

trois mois. Ils sont d'ailleurs plus nombreux à recourir aux contrats très courts (moins d'un mois).

Caractérisée par la concurrence sur les emplois vacants la plus forte et un indicateur de tension dans la moyenne, la classe des « métiers concurrentiels » est également celle où le nombre de demandeurs d'emploi par salarié est le deuxième plus élevé, le niveau moyen de l'indicateur de tension étant dès lors la conséquence d'une concurrence particulièrement intense entre les employeurs sur les emplois vacants. Elle rassemble $19 \%$ de l'emploi salarié et des recrutements.

Les métiers concernés sont plutôt des métiers qualifiés de cadres et de professions intermédiaires: professionnels de l'action culturelle, sportive et surveillants, cadres des services administratifs, comptables et financiers, cadres commerciaux et technicocommerciaux, formateurs. Sont également présents les employés de la comptabilité, les cuisiniers, les ouvriers qualifiés du second œuvre du bâtiment ainsi que les ouvriers non qualifiés de la mécanique.

Les établissements de cette classe appartiennent plus souvent aux secteurs des services non marchands et des services aux ménages ainsi qu'à celui de l'hébergement et de la restauration. Un peu plus anciens que les établissements des classes précédentes (ils existent depuis au moins dix ans au moment de l'enquête), ils appartiennent plus rarement à un groupe ou à une entreprise multi-établissements. Ils sont moins nombreux à avoir connu une croissance de leur chiffre d'affaires et/ou de leurs effectifs au cours des trois années précédant l'enquête et à avoir adopté de nouvelles techniques (nouvelles technologies de l'information et de la communication, nouveaux process, fabrication ou conception assistée par ordinateur, etc.). Leurs postes à pourvoir sont d'ailleurs, un peu plus souvent que dans les autres classes, destinés à remplacer des salariés. Ils sont un peu plus fréquemment localisés dans l'aire urbaine de Paris et recrutent davantage sur un seul poste de ce type en même temps. 
La classe des « métiers tendus fluides » présente un indicateur de tension élevé et une part anticipée de difficultés de recrutement supérieure à la moyenne, même si cette part est un peu inférieure à celle de la classe suivante. Elle se caractérise également par le taux de sortie du chômage le plus élevé, traduisant une certaine fluidité du marché du travail. Les postes vacants en concurrence semblent ainsi permettre aux demandeurs d'emploi d'obtenir plus rapidement un emploi. Un autre indicateur de cette situation est le nombre plus important d'emplois vacants par salarié, traduisant une certaine rotation sur les emplois. Cette classe représente la plus faible part de l'emploi salarié $(11 \%)$ et $14 \%$ des recrutements.

Les postes sont très majoritairement des postes de professions intermédiaires - attachés commerciaux et représentants, infirmiers et sages-femmes et professions paramédicales - et d'employés non qualifiés - employés et agents de maîtrise de l'hôtellerie et restauration. Cette classe compte également les coiffeurs et esthéticiens.

Plus de $40 \%$ des établissements qui recrutent sur des métiers de cette classe sont positionnés sur le secteur de l'hébergement et de la restauration et comptent moins de dix salariés. Ils appartiennent également plus rarement à une entreprise multiétablissements que les autres. Plus nombreux à avoir adopté de nouveaux produits ou services au cours des trois années précédant l'enquête, ils recrutent davantage en CDI et/ou pour remplacer un salarié ayant quitté temporairement ou définitivement l'établissement.

La classe des « métiers tendus difficiles » est celle où l'indicateur de tension est le plus élevé. Cette tension reflète davantage une pénurie de candidats mesurée par le nombre de demandeurs d'emploi par salarié - la plus faible des cinq classes - qu'un excès de concurrence sur les postes vacants, même si le nombre d'emplois vacants par salarié reste supérieur à la moyenne. Les employeurs de cette classe sont ceux qui anticipent les difficultés de recrutement les plus importantes. Cette classe concerne $15 \%$ de l'emploi salarié et la plus faible part des recrutements (11\%). Comme celle des «métiers concurrentiels », c'est celle où la part des recrutements passant par Pôle emploi est la plus faible.

Les emplois concernés sont globalement parmi les plus qualifiés avec ceux de la classe des «métiers concurrentiels » : postes de techniciens et agents de maîtrise de la maintenance et du bâtiment et des travaux publics, d'ingénieurs et techniciens de l'informatique et de médecins et assimilés. Sont également présents les ouvriers qualifiés du gros œuvre du bâtiment ainsi que les bouchers, charcutiers et boulangers. Les personnes qui exercent ces métiers sont moins nombreuses que les salariés des autres classes à avoir le sentiment qu'elles seront amenées à changer de métier au cours des trois prochaines années, en partie en raison de la forte tension associée à leur métier (tableau 1B en annexe). Elles sont également plus soumises à des contraintes physiques dans l'exercice de leur emploi ainsi qu'à des horaires atypiques.

Les établissements de cette classe interviennent davantage dans les secteurs de l'industrie, de la construction et de l'information et la communication. Ils comptent 
Quels liens entre tension sur le marché du travail, difficultés de recrutement et efforts de recherche des employeurs?

GRAPHIQUE 2 - Les familles professionnelles des cinq classes de métiers

\begin{tabular}{|c|c|}
\hline 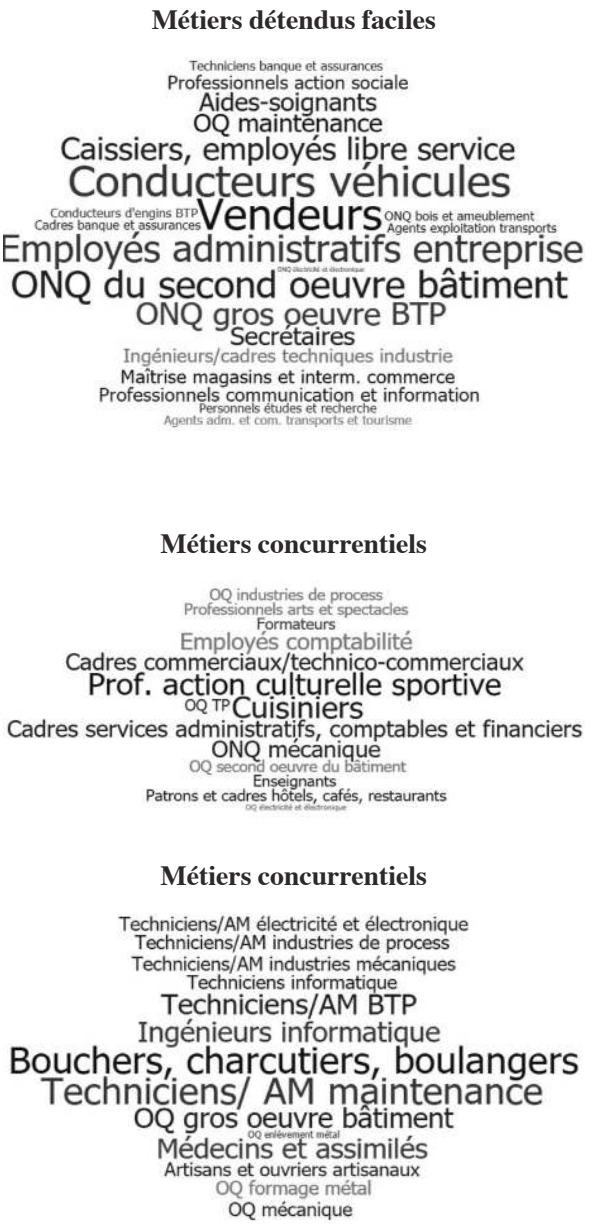 & 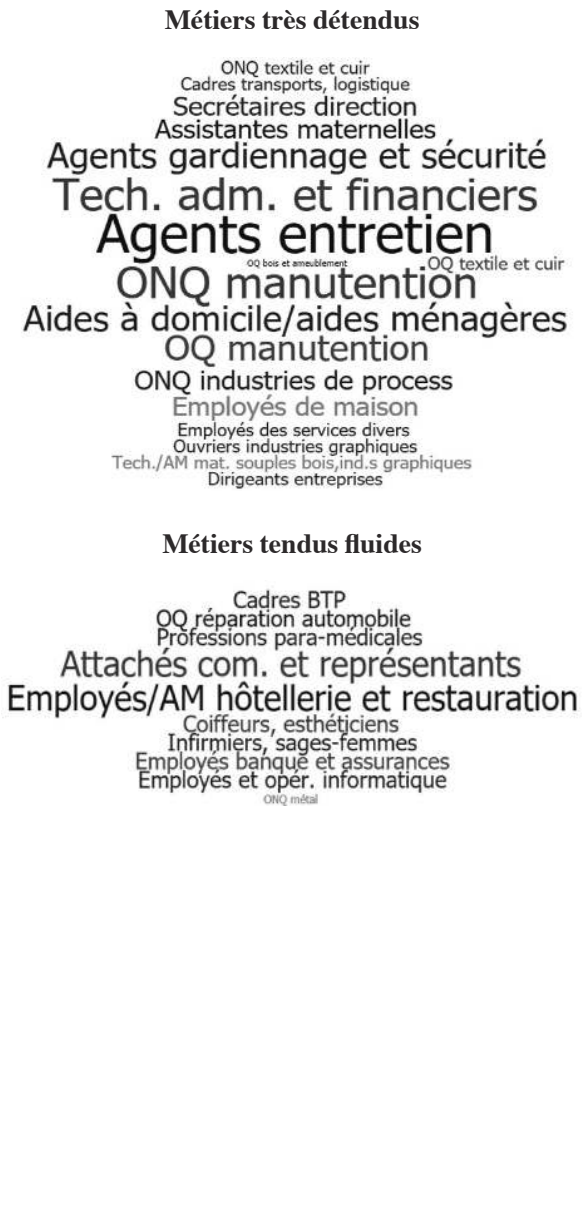 \\
\hline
\end{tabular}

OQ: ouvriers qualifiés; ONQ: ouvriers non qualifiés; AM: agents de maîtrise.

Lecture : la taille des professions est proportionnelle à leur fréquence en termes de recrutements dans la classe de métiers considérée. Champ : recrutements des établissements d'au moins un salarié du secteur concurrentiel ayant nouvellement recruté en CDI ou en CDD de plus d'un mois entre septembre et novembre 2015 en France métropolitaine à l'exception des domaines professionnels A (agriculture, marine, pêche), $\mathrm{P}$ (administration publique, professions juridiques, armée et police) et $\mathrm{X}$ (politique, religion).

Source: Dares, enquête Ofer 2016.

un peu plus souvent deux cents salariés ou plus. Ayant connu plus fréquemment une hausse de leur chiffre d'affaires au cours des trois années précédant l'enquête que les établissements des autres classes, ils sont plus nombreux à avoir innové sur cette période. Ils recrutent plus souvent que les autres sur des postes nouvellement créés, en CDD long, à temps plein. Ils sont également un peu plus nombreux que ceux des autres classes à recourir à l'intérim. 


\section{De la tension sur le métier à la difficulté du recrutement}

Cette partie vise à étudier dans quelle mesure le contexte global de tension du métier sur lequel l'établissement recrute se traduit par des difficultés de recrutement ex post. Elle permet d'interroger le lien trop souvent fait entre un nombre élevé d'emplois à pourvoir à un instant donné et les difficultés effectivement rencontrées par les employeurs lors de l'embauche. Avant d'interroger ce lien, nous allons présenter nos indicateurs de difficultés ex post.

\section{Les indicateurs de difficultés ex post}

D'après l'enquête Ofer, $17 \%$ des recrutements réalisés entre septembre et novembre 2015 sont jugés a posteriori particulièrement ou plutôt difficiles par les recruteurs. À titre de comparaison, la part des embauches anticipées comme difficiles dans l'enquête $B M O$ est de 32 \% pour l'année 2015. Par ailleurs, l'enquête Ofer 2016 s'inscrit dans un contexte macroéconomique spécifique: la part des projets de recrutement anticipés comme difficiles par les employeurs dans l'enquête $B M O$ atteint son plus bas niveau depuis 2010, le taux de chômage étant, quant à lui, à son point le plus haut (Lhommeau, Rémy, 2019b). Cette part remonte par la suite, en lien avec l'amélioration progressive de la conjoncture sur le marché du travail, le taux de chômage diminuant régulièrement.

Dans l'enquête $O f e r$, les employeurs déclarant avoir rencontré des difficultés de recrutement étaient également interrogés sur la nature de ces dernières ${ }^{16}$ (graphique 3 ). Les trois premières sources de difficultés sont l'insuffisance du nombre de candidatures reçues, le décalage entre le profil des candidats et les exigences des employeurs (en termes de diplôme, spécialité de formation, expérience, compétences techniques, qualités personnelles) et/ou celles des candidats (en matière de rémunération, conditions de travail, etc.) et les caractéristiques du poste (rémunération, difficulté ou pénibilité du travail demandé, temps de travail et/ou horaires, nature ou durée du contrat, technicité très pointue du poste). La concurrence de la part des autres entreprises est mentionnée dans $5 \%$ des recrutements et souvent en même temps que le manque de candidatures devant le déficit d'image de l'entreprise, du secteur d'activité ou du métier et les difficultés propres à l'établissement. Ces dernières recouvrent les situations de budget non garanti, de manque de temps ou de moyens pour recruter, les problèmes de coordination entre les différentes parties prenantes du recrutement, de changement de direction, etc. Cette hiérarchie des difficultés apparaît cohérente avec celle des autres sources disponibles sur cette question (l'enquête Ofer 2005 avec un questionnement et une conjoncture différents, l'enquête $B M O$ complémentaire 2015 avec le même podium). Les différentes difficultés sont très souvent mentionnées

16. Ils pouvaient choisir jusqu'à sept types de difficultés: les six items détaillés ici ainsi qu'une modalité «autre », non étudiée dans la suite de l'article car trop réduite (elle concerne moins de $1 \%$ des recrutements). 


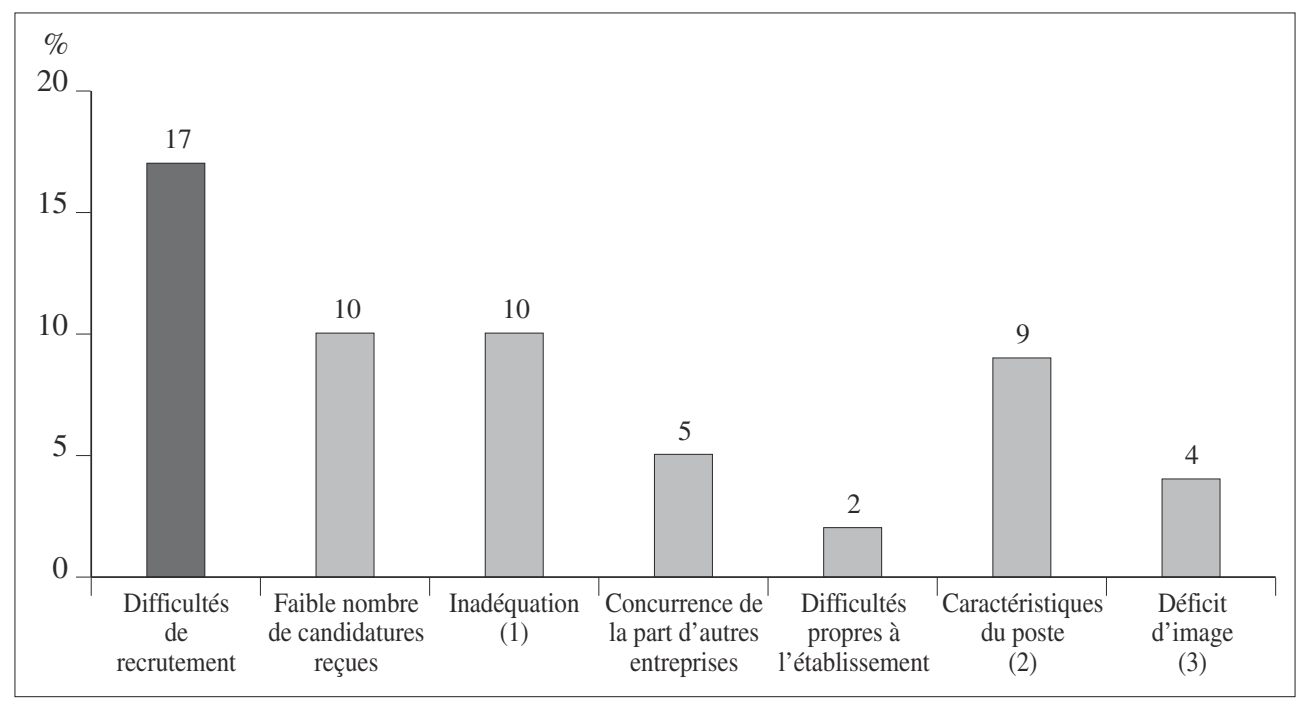

Notes : (1) Entre le profil des candidats et les attentes des recruteurs en termes d'expérience, de diplôme, de compétences techniques, de qualités personnelles, etc. (2) Rémunération, difficulté ou pénibilité du travail demandé, temps de travail et/ou horaires, nature, durée du contrat, technicité du poste. (3) De l'entreprise, du secteur d'activité ou du métier proposé.

Les employeurs pouvaient déclarer plusieurs difficultés de recrutement.

Lecture : $17 \%$ des recrutements ont été jugés très ou plutôt difficiles par les employeurs. $10 \%$ des recrutements l'ont été en raison du faible nombre de candidatures.

Champ : recrutements des établissements d'au moins un salarié du secteur concurrentiel ayant nouvellement recruté en CDI ou en CDD de plus d'un mois entre septembre et novembre 2015 en France métropolitaine à l'exception des domaines professionnels A (agriculture, marine, pêche), $\mathrm{P}$ (administration publique, professions juridiques, armée et police) et $\mathrm{X}$ (politique, religion).

Source: Dares, enquête Ofer 2016.

simultanément puisque seuls $25 \%$ des recruteurs rencontrant des difficultés n'en citent qu'une seule. La nature des difficultés rencontrées varie fortement selon les familles professionnelles.

Nous utilisons également deux indicateurs complémentaires de la difficulté de recrutement, que l'on peut considérer comme plus objectifs et qui sont également mesurés à partir d'Ofer: le temps que l'employeur avait prévu de consacrer au recrutement ainsi que le temps qu'il y a effectivement dédié. En effet, dans une précédente étude (LHOMMEAU, RÉMY, 2019b), nous avons montré que la complexité du recrutement a été en partie anticipée par les recruteurs puisqu'en cas de difficulté avérée, ces derniers envisageaient déjà d'y consacrer un temps plus long.

\section{Quels effets du contexte global de tension sur les difficultés ex post?}

Dans quelle mesure le contexte global de tension associé à telle ou telle classe de métier (métiers détendus faciles, très détendus, concurrentiels, tendus fluides ou tendus difficiles) est-il corrélé, toutes choses égales par ailleurs, au fait que l'employeur déclare avoir rencontré des difficultés de recrutement ex post ou encore qu'il mentionne 
tel ou tel type de difficultés (manque de candidatures, concurrence de la part des autres recruteurs, etc.) ? Une modélisation de type probit permet de répondre à cette question ${ }^{17}$.

Nos résultats montrent que recruter dans un contexte global de tension élevée n'est pas systématiquement plus difficile que dans un contexte global de tension faible. Certes, le lien entre contexte global de tension élevée et difficulté de recrutement ex post se vérifie pour la classe des «métiers tendus difficiles » (tableau 2). Les établissements de cette classe, confrontés à la tension la plus élevée et déclarant la part d'embauches anticipées comme difficiles la plus importante, font plus souvent état de difficultés de recrutement que ceux de la classe des «métiers détendus faciles » à caractéristiques du poste et de l'établissement comparables. Appartenir à la classe des «métiers tendus difficiles » n'est cependant pas associé à un temps prévu pour recruter plus important ou une durée effective plus longue que pour la classe de référence (tableau 3).

De manière plus surprenante, les employeurs de la classe des «métiers très détendus » sont, toutes choses égales par ailleurs, plus nombreux à avoir déclaré des difficultés de recrutement que ceux de la classe des «métiers détendus faciles » (tableau 2). Si les employeurs de la première classe anticipaient un peu plus de difficultés que ceux de la seconde (avec une part de projets de recrutement anticipés comme difficiles de $35 \%$ contre $28 \%$ ), ils recrutent pourtant sur un marché du travail très peu tendu $^{18}$ (tableau 1). Ces difficultés ex post ne semblent pas se traduire par un allongement du processus de recrutement puisque ce dernier a été parmi les plus rapides, et tiennent probablement davantage à l'attractivité du poste à pourvoir. Les recruteurs de cette classe sont en effet plus nombreux à mettre en cause les caractéristiques du poste à pourvoir - en termes de rémunération, de conditions de travail, etc. - comme source de difficultés de recrutement. Les métiers de cette classe sont d'ailleurs associés à des conditions de travail plus difficiles d'après les indicateurs présentés plus haut.

De leur côté, les recruteurs de la classe des «métiers tendus fluides », pourtant confrontés à une tension élevée, ne mentionnent pas davantage de difficultés que les autres et ont même pourvu leurs postes plus rapidement.

Enfin, les employeurs de la classe des «métiers concurrentiels » avaient plus souvent que les autres (à l'exception de ceux de la classe «métiers tendus difficiles ») prévu de consacrer plus d'un mois au recrutement, même s'ils ne diffèrent pas des autres employeurs ex post en termes de difficultés ou de durée de l'embauche

\footnotetext{
17. La modélisation isole l'effet du contexte global de tension sur la probabilité que le recruteur mentionne des difficultés de recrutement ex post en contrôlant d'autres facteurs susceptibles d'affecter cette probabilité: les caractéristiques des établissements et celles des emplois sur lesquels ont porté leurs recrutements. Plus précisément, ont été intégrées dans les estimations les caractéristiques suivantes: le secteur d'activité de l'établissement, sa taille, sa catégorie juridique, sa localisation, son ancienneté, son caractère mono ou multi-établissements, son appartenance à un groupe, le caractère unique ou multiple du recrutement, la qualification du poste à pourvoir, le type de contrat, son caractère subventionné ou non et le temps de travail associé.

18. Ils n'avaient d'ailleurs pas prévu de consacrer davantage de temps au recrutement que les autres puisqu'ils sont moins nombreux à déclarer avoir disposé d'une durée supérieure à trente jours pour recruter.
} 
TABLEAU 2 - Difficultés de recrutement selon les cinq classes de métiers (effets marginaux)

\begin{tabular}{|c|c|c|c|c|c|c|c|}
\hline & $\begin{array}{c}\text { Difficultés } \\
\text { de recru- } \\
\text { tement }\end{array}$ & $\begin{array}{c}\text { Faible } \\
\text { nombre } \\
\text { de candi- } \\
\text { datures } \\
\text { reçues }\end{array}$ & $\begin{array}{c}\text { Inadéqua- } \\
\text { tion } \\
\text { (1) }\end{array}$ & $\begin{array}{c}\text { Concur- } \\
\text { rence de } \\
\text { la part } \\
\text { d'autres } \\
\text { entreprises }\end{array}$ & $\begin{array}{c}\text { Difficultés } \\
\text { propres à } \\
\text { l'établisse- } \\
\text { ment }\end{array}$ & $\begin{array}{l}\text { Caracté- } \\
\text { ristiques } \\
\text { du poste } \\
(2)\end{array}$ & $\begin{array}{c}\text { Déficit } \\
\text { d'image } \\
\text { (3) }\end{array}$ \\
\hline Métiers très détendus & $0,04 * * *$ & $0,02 *$ & 0,02 & 0,01 & 0,01 & $0,02 * *$ & $0,02 * * *$ \\
\hline Métiers concurrentiels & 0,02 & $0,02 * *$ & 0,00 & $0,02 * *$ & 0,00 & 0,00 & $0,01 *$ \\
\hline Métiers tendus fluides & 0,01 & $0,03 * *$ & $-0,01$ & 0,01 & 0,00 & $-0,01$ & $0,02 * *$ \\
\hline Métiers tendus difficiles & $0,03 *$ & $0,05 * * *$ & 0,00 & $0,02 * * *$ & $-0,01 *$ & 0,01 & $0,03 * * *$ \\
\hline $\begin{array}{l}\text { Métiers détendus faciles } \\
\text { (réf.) }\end{array}$ & & & & & & & \\
\hline
\end{tabular}

Notes : (1) Entre le profil des candidats et les attentes des recruteurs en termes d'expérience, de diplôme, de compétences techniques, de qualités personnelles, etc. (2) Rémunération, difficulté ou pénibilité du travail demandé, temps de travail et/ou horaires, nature, durée du contrat, technicité du poste. (3) De l'entreprise, du secteur d'activité ou du métier proposé.

Ne sont ici présentés que les résultats concernant les effets marginaux de l'appartenance à une classe donnée de la typologie. Les modèles estimés sont des probit simples qui comprennent également comme variables de contrôle: le secteur d'activité de l'établissement, sa taille, sa catégorie juridique, sa localisation, son ancienneté, son caractère mono ou multi-établissements, son appartenance à un groupe, le caractère unique ou multiple du recrutement, la qualification du poste, le type de contrat, son caractère subventionné ou non et le temps de travail associé. Les symboles *,** et *** représentent les seuils de significativité statistique à 10,5 et $1 \%$.

Lecture : le fait que l'employeur appartienne à la classe des « métiers très détendus » plutôt qu'à celle des « métiers détendus faciles » augmente de 4 points de \% la probabilité qu'il déclare avoir rencontré des difficultés ex post pour recruter.

Champ : recrutements des établissements d'au moins un salarié du secteur concurrentiel ayant nouvellement recruté en CDI ou en CDD de plus d'un mois entre septembre et novembre 2015 hors domaines professionnels A (agriculture, marine, pêche), P (administration publique, professions juridiques, armée et police) et X (politique, religion); France métropolitaine.

Source : Dares, enquête Ofer 2016.

(tableaux 2 et 3). Ce comportement peut s'expliquer par la forte concurrence mesurée par le nombre d'emplois vacants par salarié à laquelle ils doivent faire face.

Seuls certains types de difficultés rencontrées par les recruteurs sont susceptibles de varier avec le contexte global de tension. C'est le cas des difficultés dues à un nombre insuffisant de candidatures : les établissements des classes des « métiers tendus difficiles » et des « métiers tendus fluides » évoquent plus que ceux des autres classes un manque de candidats, en lien avec l'indicateur de tension élevé auquel ils sont confrontés (tableaux 1 et 2). À l'opposé, les employeurs de la classe des «métiers détendus faciles » font moins souvent mention de cette pénurie de candidatures. Ils ont d'ailleurs examiné un plus grand nombre de candidatures que ceux des autres classes (tableau 3). Ils sont moins nombreux que les autres recruteurs à déplorer le déficit d'image du métier, du secteur d'activité ou de l'établissement (tableau 2).

De leur côté, les établissements des classes des « métiers concurrentiels » et des « métiers tendus difficiles » sont plus nombreux à faire état de la concurrence de la part des autres entreprises du secteur (tableau 2). Même si les premiers ont un indicateur de tension dans la moyenne, le nombre d'emplois vacants par salarié est le plus élevé, traduisant une concurrence sur le métier concerné. Les seconds recrutent sur les marchés où l'indicateur de tension est le plus élevé, cet indicateur de tension élevé étant plutôt attribuable à une pénurie de candidats potentiels qu'à une concurrence entre candidats, concurrence qui, certes est au-dessus de la moyenne mais comparable à celle observée dans la classe des « métiers tendus fluides ». 
TABLEAU 3 - Durée prévue et effective du recrutement et nombre de candidatures examinées

\begin{tabular}{lccc}
\hline & $\begin{array}{c}\text { Plus de 30 jours } \\
\text { prévus pour recruter }\end{array}$ & $\begin{array}{c}\text { Durée } \\
\text { du recrutement } \\
(\mathbf{1})\end{array}$ & $\begin{array}{c}\text { Nombre de } \\
\text { candidatures examinées } \\
\mathbf{( 2 )}\end{array}$ \\
\hline Métiers très détendus & $-0,06 * * *$ & $-0,20 * * *$ & $-0,07 *$ \\
Métiers concurrentiels & $0,03 * *$ & $-0,01$ & $-0,26 * * *$ \\
Métiers tendus fluides & $-0,01$ & $-0,13 * * *$ & $-0,17 * * *$ \\
Métiers tendus difficiles & 0,02 & 0,00 & $-0,28 * * *$ \\
Métiers détendus faciles (réf.) & & & \\
\hline
\end{tabular}

Notes: (1) Sur le champ des établissements en mesure d'identifier la durée du processus de recrutement. (2) Sur le champ des établissements en mesure de dénombrer le nombre de candidatures examinées.

Ne sont ici présentés que les résultats concernant l'appartenance à une classe donnée de la typologie. Les modèles estimés sont des probit ordonnés pour la durée (répartie en 3 tranches: moins de 8 jours, 8 à 30 jours, plus de 30 jours) et le nombre de candidatures examinées (réparti en 4 tranches: 1, 2 à 4, 5 à 9, 10 et plus), et un probit simple avec calcul d'effets marginaux pour la durée prévue. Ils comprennent également les variables de contrôle citées en note du tableau 2 . Les symboles *, ** et *** représentent les seuils de significativité statistique à 10,5 et $1 \%$.

Lecture : le fait que l'employeur appartienne à la classe des « métiers très détendus » plutôt qu'à celle des « métiers détendus faciles » diminue de 6 points de $\%$ la probabilité qu'il déclare avoir prévu de consacrer plus de 30 jours au recrutement.

Champ : recrutements des établissements d'au moins un salarié du secteur concurrentiel ayant nouvellement recruté en CDI ou en CDD de plus d'un mois entre septembre et novembre 2015 hors domaines professionnels A (agriculture, marine, pêche), P (administration publique, professions juridiques, armée et police) et $\mathrm{X}$ (politique, religion); France métropolitaine.

Source: Dares, enquête Ofer 2016.

A contrario, les établissements de la classe des « métiers tendus difficiles » font moins souvent état de difficultés propres à l'établissement (manque de temps, de moyens pour recruter, etc. $)^{19}$, cela pouvant en partie s'expliquer par l'organisation du processus de recrutement dans cette classe détaillée dans la section suivante. Les différentes classes ne diffèrent pas au regard de l'inadéquation entre les profils des candidats et les exigences des recruteurs, cette difficulté semblant davantage relever de la qualification du poste ou du manque d'habitude de recruter de l'établissement (Lhommeau, Rémy, 2019a) que du contexte global de tension prévalant sur le métier considéré.

En conclusion, un contexte global de tension élevée ne s'accompagne pas systématiquement de difficultés de recrutement ex post: parmi les recruteurs des deux classes de métiers les plus tendus, seuls ceux de la classe des «métiers tendus difficiles » font état de difficultés. Néanmoins, un tel contexte augmente les chances que les employeurs déclarent manquer de candidatures, souffrir d'un déficit d'image et dans certains cas, pâtir de la concurrence d'autres entreprises.

19. Ces difficultés touchent néanmoins globalement peu les recruteurs: seuls $2 \%$ de l'ensemble des recrutements sont concernés. 


\section{De la tension sur le métier aux moyens mis en œuvre}

On pourrait s'attendre à ce que les moyens mobilisés par les employeurs pour recruter - les canaux activés pour rechercher des candidats, les méthodes pour les sélectionner, les concessions que sont prêts à faire les employeurs ou encore l'organisation de leur processus de recrutement - soient plus conséquents dans un contexte global de tension élevée. Avant de vérifier si cette intuition est fondée, nous présentons d'abord les principaux indicateurs retenus pour mesurer les efforts de recrutement des employeurs.

\section{Les indicateurs d'effort de recrutement}

On peut approcher les efforts consacrés au recrutement par les employeurs à partir des quatre grandes catégories de méthodes qu' ils peuvent mobiliser pour accroître leur intensité de recrutement (FABERMAN, 2014): offrir des salaires supérieurs au salaire de marché pour attirer plus de candidats et qu'ils aient plus de chances d'accepter le poste proposé, étendre les recherches de candidats en utilisant leur réseau de relations ou en diffusant largement leurs offres d'emploi, réduire leurs exigences en matière d'embauche, ou encore accroître leur effort de sélection afin de s'assurer que suffisamment de candidats de qualité soient reçus en entretien. L'enquête Ofer 2016 ne nous permet pas de mesurer précisément la première catégorie de méthodes ${ }^{20}$ mais nous pouvons construire des indicateurs pour les trois autres.

Pour mesurer l'étendue de la recherche des recruteurs, nous retenons le nombre de canaux de recherche de candidats mobilisés (regroupés en cinq grandes catégories, présentées dans le graphique 4) ainsi que la nature de ces canaux en allant du canal le plus intensif, les relations, au plus extensif, les annonces. Les premières permettent aux recruteurs d'avoir une information de qualité sur un nombre restreint de candidats tandis que les dernières leur offrent la possibilité de toucher un grand nombre de candidats, mais avec une information limitée sur chacun d'entre eux (REES, SHULTZ, 1970; DeVaro, 2008). Le graphique 4 indique le taux de recours des recruteurs aux différents canaux. Les recruteurs ont mobilisé en moyenne 2,5 canaux pour mener à bien leurs recherches de candidats (nombre calculé sur les cinq grandes catégories). Les canaux les plus souvent utilisés sont l'examen de candidatures spontanées et les relations, sollicités chacun dans plus de six recrutements sur dix. Cette fréquence peut s'expliquer par le faible coût de l'activation des candidatures spontanées et la réduction d'incertitude sur les candidats grâce aux relations (REES, 1966; DEVARO, 2005). La diffusion d'annonces est un peu moins répandue, les parts respectives des intermédiaires publics - Pôle emploi, mission locale ou autre service public de l'emploi

20. Et ce malgré la présence dans l'enquête de deux indicateurs indirects d'un éventuel ajustement des salaires proposés que nous utilisons ponctuellement dans notre analyse. Une question est ainsi posée à l'employeur sur le fait qu'il se soit enquis du niveau de salaire ou des prétentions salariales des candidats. Par ailleurs, lorsqu'il fait état de difficultés de recrutement, nous savons si l'employeur a ajusté les caractéristiques du poste, dont la rémunération. 


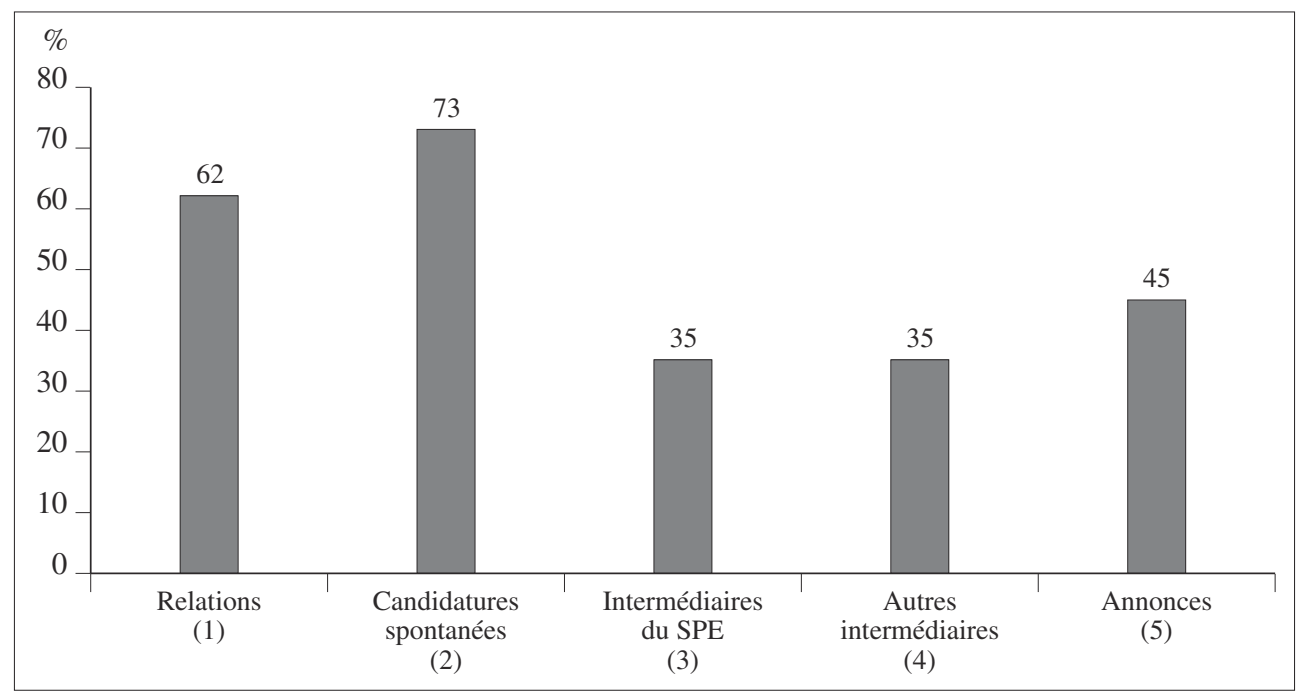

Notes: (1) Sont ici regroupés les relations personnelles et professionnelles et le rappel de personnes ayant déjà travaillé pour l'établissement. (2) Est également incluse la consultation de bases de CV internes (candidatures reçues sur un précédent poste ou base de profils constituée par l'établissement) ou de bases de CV externes. (3) Pôle emploi, missions locales et autres intermédiaires du service public de l'emploi. (4) Cette catégorie comprend l'Agence pour l'emploi des cadres (Apec), les organismes professionnels ou groupements d'employeurs, les écoles, universités et centres de formation, les cabinets de recrutement, les agences d'intérim ou agences d'emploi, les opérateurs privés de placement et les autres organismes. (5) La participation à des salons et les autres canaux ont été regroupés avec les annonces.

Lecture: les employeurs mobilisent leurs relations pour $62 \%$ de leurs recrutements.

Champ : recrutements des établissements d'au moins un salarié du secteur concurrentiel ayant nouvellement recruté en CDI ou en CDD de plus d'un mois entre septembre et novembre 2015 en France métropolitaine à l'exception des domaines professionnels A (agriculture, marine, pêche), $\mathrm{P}$ (administration publique, professions juridiques, armée et police) et $\mathrm{X}$ (politique, religion).

Source: Dares, enquête Ofer 2016.

(SPE) - et des « autres intermédiaires » (écoles ou centres de formation, cabinets de recrutement, entreprises d'intérim, etc.) étant proches: un peu plus d'un tiers des embauches. Cette hiérarchie dans l'usage des canaux de recrutement est comparable à celle observée dans des travaux français antérieurs conduits sur les établissements (BESSY, MARCHAL, 2009).

Concernant l'assouplissement des exigences, nous utilisons les questions sur les attentes des recruteurs en matière de diplôme ainsi que sur les arbitrages entre diplôme et expérience et leur propension à embaucher un chômeur. Interrogés sur leurs attentes en matière de diplôme, $35 \%$ des recruteurs déclarent ne pas en avoir. Mais leurs réponses sont très variables selon la Fap considérée: par exemple, $70 \%$ des recruteurs sur un poste d'ouvrier non qualifié de la manutention n'ont aucune attente en la matière tandis que la quasi-totalité de ceux cherchant à pourvoir des emplois d'infirmiers et de sages-femmes en ont. Concernant les arbitrages entre diplôme et expérience, 73 \% des recruteurs auraient pu embaucher une personne moins diplômée mais plus expérimentée et, inversement, $41 \%$ étaient prêts à recruter une personne moins expérimentée mais plus diplômée. Enfin, interrogés sur leur propension à embaucher un chômeur à 
compétences égales, $24 \%$ des employeurs déclarent qu'ils auraient pu en recruter un, à condition que son épisode de chômage ne soit pas trop long, 62 \% quelle que soit cette durée et enfin, $15 \%$ ne l'auraient pas fait. Par ailleurs, $35 \%$ des postes ont été pourvus avec une personne qui était au chômage avant son entrée dans l'établissement.

Du point de vue de l'effort de sélection, nous avons retenu trois indicateurs principaux: le nombre de pièces demandées à l'ensemble ou une partie des candidats, le nombre de méthodes de sélection mobilisées ainsi que la part des candidats reçus en entretien individuel. Les pièces demandées sont les suivantes : un $\mathrm{CV}$, les nom et adresse des candidats, une lettre de motivation, des certificats ou autres documents administratifs, le niveau de salaire ou les prétentions salariales, une copie du diplôme ou une attestation de formation, un dossier de candidature rempli, le permis de conduire, des références ou recommandations, une photo d'identité ou un autre document. Les méthodes de sélection regroupent quant à elles les entretiens individuels, les entretiens téléphoniques, les mises à l'essai, les tests reproduisant des situations de travail, les tests sur les compétences de base, les tests de connaissance ou d'intelligence, les tests de personnalité, les tests de langues étrangères, les épreuves de groupe ou les analyses graphologiques $^{21}$. En moyenne, les recruteurs ont demandé 4,7 éléments aux candidats et ont mis en œuvre 2,1 méthodes de sélection lors de l'embauche. Dans $27 \%$ des recrutements, moins d'un quart des candidats ont été reçus en entretien individuel, dans $33 \%$ des recrutements, entre un quart et trois quarts d'entre eux et, dans $40 \%$ des recrutements, plus de trois quarts d'entre eux. Nous allons maintenant voir dans quelle mesure les efforts des recruteurs - approchés par ces trois catégories de méthodes, à savoir l'étendue de leur recherche de candidats, l'assouplissement de leurs exigences et leur effort de sélection - sont susceptibles de varier ou non selon le contexte du marché du travail dans lequel ils interviennent.

\section{Comment le contexte global de tension affecte-t-il les canaux mobilisés pour rechercher des candidats?}

Un contexte global de tension élevée ne se traduit pas par un plus grand nombre de canaux mobilisés pour rechercher des candidats et dès lors, un effort de recherche plus important de la part de l'employeur. Il se traduit plutôt par le choix de canaux particuliers (tableau 4). Ainsi, pour faire face respectivement à la tension élevée prévalant sur les métiers qu'ils recherchent et à la concurrence des autres employeurs, les recruteurs des classes des «métiers tendus difficiles » et des « métiers concurrentiels » ont davantage sollicité les intermédiaires autres que le SPE, même si le recours à leurs services représente parfois un coût financier important: en effet, en raison de leur spécialisation sur certains segments de marché (BESSY, LARQUIER, 2010; SABATIER, 2010), ils permettent d'identifier des candidats difficiles à atteindre par les employeurs. Ce résultat confirme celui de Maxime Bergeat et Véronique Rémy (2019), qui

21. Les pièces demandées et les méthodes de sélection sont ici classées par ordre décroissant d'utilisation lors des recrutements (pour davantage de détails, voir BERGEAT, RÉMY, 2017). 
TABLEAU 4 - Canaux de recherche de candidats mobilisés selon les cinq classes de métiers

\begin{tabular}{lcccccc}
\hline & $\begin{array}{c}\text { Nombre } \\
\text { de canaux } \\
\text { mobilisés }\end{array}$ & $\begin{array}{c}\text { Relations } \\
\mathbf{( 1 )}\end{array}$ & $\begin{array}{c}\text { Candida- } \\
\text { tures } \\
\text { spontanées } \\
\mathbf{( 2 )}\end{array}$ & $\begin{array}{c}\text { Intermé- } \\
\text { diaires } \\
\text { du SPE (3) }\end{array}$ & $\begin{array}{c}\text { Autres } \\
\text { intermé- } \\
\text { diaires (4) }\end{array}$ & $\begin{array}{c}\text { Annonces } \\
\text { (5) }\end{array}$ \\
\hline Métiers très détendus & 0,02 & 0,00 & $-0,02$ & $0,05 * * *$ & $-0,01$ & $-0,01$ \\
Métiers concurrentiels & $-0,03$ & $0,05 * * *$ & $-0,04 * *$ & $-0,02$ & $0,03 * *$ & $-0,06 * * *$ \\
Métiers tendus fluides & 0,05 & $-0,01$ & $0,04 * *$ & 0,01 & 0,02 & 0,00 \\
Métiers tendus difficiles & 0,03 & 0,00 & $-0,01$ & 0,00 & $0,04 * *$ & 0,01 \\
Métiers détendus faciles (réf.) & & & & & & \\
\hline
\end{tabular}

Notes : les différents canaux de recherche mobilisés sont définis en notes du graphique 4.

Ne sont ici présentés que les résultats concernant l'appartenance à une classe donnée de la typologie (effets marginaux sauf pour le nombre de canaux mobilisés). Les modèles estimés sont des probit simples avec calcul d'effets marginaux sauf dans le cas du nombre de canaux mobilisés où il s'agit d'un probit ordonné. Ces modèles comprennent également les variables de contrôle citées en note du tableau 2. Les symboles *, ** et *** représentent les seuils de significativité statistique à 10,5 et $1 \%$.

Lecture : le fait d'appartenir à la classe des «métiers très détendus » plutôt qu'à celle des «métiers détendus faciles » augmente de 5 points de \% la probabilité d'avoir mobilisé les intermédiaires du SPE lors du recrutement.

Champ : recrutements des établissements d'au moins un salarié du secteur concurrentiel ayant nouvellement recruté en CDI ou en CDD de plus d'un mois entre septembre et novembre 2015 hors domaines professionnels A (agriculture, marine, pêche), P (administration publique, professions juridiques, armée et police) et X (politique, religion); France métropolitaine.

Source: Dares, enquête Ofer 2016.

constataient des embauches plus fréquentes via les « autres intermédiaires » en cas de tension élevée et/ou de concurrence accrue entre employeurs, même si l'analyse présentée ici porte sur le rôle de ces intermédiaires en tant que canal de recherche de candidats et non de recrutement. En revanche, contrairement à Giovanni Russo et ses co-auteurs (2000), nous ne constatons pas de recours accru aux annonces en cas de forte tension sur le marché du travail.

Les employeurs de la classe des « métiers concurrentiels » ont davantage mobilisé leurs relations qui, conformément à la littérature, sont plus à même de les orienter vers des candidats répondant à leurs attentes (REES, 1966; MARSDEN, 1988; Russo et al., 1997 ; DeVAro, 2005). De plus, en cas d'appel aux relations, les différentes étapes de sélection des candidats peuvent être réduites, une présélection ayant été réalisée par les personnes qui recommandent les candidats (MARSDEN, GORMAN, 2001). L'activité de présélection réalisée par les « autres intermédiaires » et par les relations peut d'ailleurs expliquer que les recruteurs de la classe des « métiers concurrentiels » privilégient ces canaux et recourent moins aux annonces ou aux candidatures spontanées, qui, dans les deux cas, nécessitent un tri en interne des candidatures. De leur côté, les établissements de la classe des « métiers très détendus » ont plus souvent utilisé les services du SPE, un mode de recherche de candidats peu coûteux au regard d'autres intermédiaires du marché du travail, mais permettant de toucher un vivier important de candidats. Ce résultat va dans le sens des travaux de M. BERGEAT et V. RÉMY (2019) selon lesquels le recours au SPE pour recruter est plus fréquent lorsque le taux de chômage local est élevé. En effet, la classe des «métiers très détendus » présente le nombre de demandeurs d'emploi par salarié le plus conséquent (tableau 1). 
Pour leur part, malgré la tension élevée à laquelle ils sont confrontés, les recruteurs de la classe des «métiers tendus fluides » ont mobilisé un canal peu coûteux à activer, à savoir les candidatures spontanées. Parmi les établissements des trois classes ayant les contextes globaux de tensions les plus élevées (« métiers tendus difficiles », « métiers tendus fluides » et «métiers concurrentiels »), ceux confrontés à des difficultés de recrutement sont plus nombreux à déclarer avoir intensifié leur effort afin de faciliter l'embauche, en sollicitant notamment plus intensément leurs canaux usuels de recherche de candidats. Ce résultat est cohérent avec celui de Giovanni Russo et ses co-auteurs (2000) qui mettent en évidence une relation positive entre durée de l'embauche, et difficulté potentielle de cette dernière, et effort de recrutement mesuré par le nombre de canaux activés.

\section{Le processus de sélection des candidatures varie-t-il selon le contexte global de tension?}

D'après les travaux d'A. GAVAZZA et ses co-auteurs (2018), on peut s'attendre à ce que les efforts de recrutement des employeurs, notamment lors de la phase de sélection des candidatures, soient plus importants dans un contexte global de tension élevée, et inversement plus faibles dans un contexte de tension faible. Ce lien s'observe pour le nombre d'éléments demandés aux candidats, qui est le plus faible dans la classe des « métiers détendus faciles » (tableau 5). Concernant la nature des éléments demandés, les établissements des classes des «métiers très détendus » ainsi que des «métiers concurrentiels » ont été plus nombreux que les établissements de la classe des « métiers détendus faciles » à souhaiter disposer de références ou de recommandations, ce souhait pouvant résulter de la nature des canaux de recherche de candidats activés. Les recruteurs de ces deux classes ont en effet plus souvent fait appel aux intermédiaires du marché du travail - SPE pour les premiers et « autres intermédiaires » pour les seconds. Même si ces intermédiaires ont pu effectuer une présélection parmi les candidatures, les recruteurs peuvent avoir besoin d'éléments complémentaires pour vérifier l'adéquation du profil des candidats avec le poste proposé. Les employeurs de la classe des «métiers concurrentiels » ont également plus souvent interrogé les postulants sur leur niveau de salaire ou leurs prétentions salariales que les employeurs des autres classes à l'exception de celle des « métiers tendus difficiles ». Cette demande peut s'expliquer par la forte concurrence à laquelle ils sont confrontés de la part des autres employeurs mais aussi par leur manque d'habitude de recruter - ce sont les moins nombreux à avoir recruté au moins une fois au cours de l'année passée : ils ont peut-être souhaité disposer de cette information afin d'ajuster leur offre d'emploi en conséquence.

En revanche, le lien entre contexte global de tension et effort de recrutement mesuré par le nombre de méthodes de sélection mobilisées est moins net: seuls les recruteurs de la classe des « métiers concurrentiels » se distinguent par le faible nombre de méthodes mobilisées. Les recruteurs se différencient, en revanche, sur la part des 
TABLEAU 5 - Pièces demandées et méthodes de sélection selon les cinq classes de métiers

\begin{tabular}{lccccc}
\hline & $\begin{array}{c}\text { Nombre } \\
\text { d'éléments } \\
\text { demandés }\end{array}$ & $\begin{array}{c}\text { Références ou } \\
\text { recomman- } \\
\text { dations }\end{array}$ & $\begin{array}{c}\text { Niveau de } \\
\text { salaire ou } \\
\text { prétentions } \\
\text { salariales }\end{array}$ & $\begin{array}{c}\text { Nombre } \\
\text { de méthodes } \\
\text { de sélection }\end{array}$ & $\begin{array}{c}\text { Part des } \\
\text { candidats reçus } \\
\text { en entretien } \\
\text { individuel }\end{array}$ \\
\hline Métiers très détendus & $0,10 * * *$ & $0,05 * * *$ & $-0,03 *$ & 0,05 & $-0,06 *$ \\
Métiers concurrentiels & $0,12 * * *$ & $0,03 * *$ & $0,05 * * *$ & $-0,07 *$ & 0,02 \\
Métiers tendus fluides & $0,09 * *$ & 0,01 & $-0,04 * *$ & $-0,04$ & $0,12 * *$ \\
Métiers tendus difficiles & $0,13 * * *$ & 0,02 & 0,03 & 0,00 & $0,15 * * *$ \\
Métiers détendus faciles (réf.) & & & & & \\
\hline
\end{tabular}

Notes : les méthodes de sélection ont pu être mobilisées pour certains ou l'ensemble des candidats. Les éléments ont pu être demandés à certains ou à l'ensemble des candidats.

Ne sont ici présentés que les résultats concernant l'appartenance à une classe donnée de la typologie. Les modèles estimés sont des probit ordonnés pour le nombre d'éléments demandés (répartis en 3 tranches : 3 pièces ou moins, 4 à 5 pièces, 6 pièces ou plus), le nombre de méthodes de sélection (réparties en 4 tranches: aucune, 1, 2, 3 ou plus) et la part des candidats reçus en entretien individuel (répartie en 3 tranches : $25 \%$ ou moins, entre $25 \%$ et $75 \%$, plus de $75 \%$ ), et des probit simples avec calcul d'effets marginaux pour les autres variables. Ils comprennent également les variables de contrôle citées en note du tableau 2 . Les symboles *, ** et *** représentent les seuils de significativité statistique à 10,5 et $1 \%$.

Lecture: le fait d'appartenir à la classe des « métiers très détendus » plutôt qu'à celle des « métiers détendus faciles » augmente de 5 points de $\%$ la probabilité d'avoir demandé des références ou des recommandations à certains ou à l'ensemble des candidats.

Champ : recrutements des établissements d'au moins un salarié du secteur concurrentiel ayant nouvellement recruté en CDI ou en CDD de plus d'un mois entre septembre et novembre 2015 hors domaines professionnels A (agriculture, marine, pêche), P (administration publique, professions juridiques, armée et police) et X (politique, religion); France métropolitaine.

Source: Dares, enquête Ofer 2016.

candidats reçus en entretien individuel: cette part est plus importante dans les deux classes de métiers les plus tendus («métiers tendus difficiles » et «métiers tendus fluides » $)^{22}$ alors qu'elle est plus faible dans celle des «métiers très détendus » que dans celle des «métiers détendus faciles » (tableau 5). Les recruteurs de la classe des «métiers très détendus » semblent privilégier différents types de tests - vérification des compétences de base (lire, écrire, compter), tests de connaissances et d'intelligence, de personnalité, reproduisant des situations de travail - pour évaluer les candidats.

Les recruteurs des trois classes de métiers les plus tendus sont également moins équipés pour recruter (tableau 6) : ils sont moins nombreux à disposer d'un service des ressources humaines $(\mathrm{RH})$ que ceux de la classe des «métiers détendus faciles ». Par ailleurs, les établissements des classes « métiers tendus difficiles » et « métiers concurrentiels » sont moins nombreux que les établissements des autres classes de métiers à disposer d'un cadre formalisé, puisque la procédure de recrutement y est moins souvent définie selon la catégorie du poste à pourvoir. Dans la classe des «métiers tendus fluides », l'absence d'un service RH s'accompagne d'une implication plus fréquente du directeur de l'établissement dans la procédure de recrutement, contrairement à la classe des «métiers très détendus » dans laquelle cette implication est plus rare. Ces deux classes sont celles où le recrutement est le plus souvent le fait d'une seule personne. Le coût interne du recrutement - mesuré par le temps consacré à l'embauche par le personnel en charge du recrutement - y est également le plus limité; le fait qu'il

22. Les recruteurs de ces deux classes ont également été moins nombreux à éliminer des candidats sans contact direct. 
Quels liens entre tension sur le marché du travail, difficultés de recrutement

et efforts de recherche des employeurs?

TABLEAU 6 - Organisation du recrutement selon les cinq classes de métiers

\begin{tabular}{|c|c|c|c|c|c|}
\hline & \multirow[t]{2}{*}{$\begin{array}{c}\text { Présence } \\
\text { d'un service } \\
\text { RH }\end{array}$} & \multicolumn{2}{|c|}{$\begin{array}{c}\text { Nombre de personnes } \\
\text { impliquées dans le recrutement } \\
\text { (réf: deux) }\end{array}$} & \multirow[t]{2}{*}{$\begin{array}{c}\text { Implication } \\
\text { du chef } \\
\text { d'établissement }\end{array}$} & \multirow[t]{2}{*}{$\begin{array}{l}\text { Coût interne du } \\
\text { recrutement }\end{array}$} \\
\hline & & Plus de deux & Une seule & & \\
\hline Métiers très détendus & $-0,01$ & $-0,09$ & $0,15 *$ & $-0,05 * * *$ & $-0,09 * * *$ \\
\hline Métiers concurrentiels & $-0,02 *$ & $0,17 *$ & 0,09 & 0,02 & $-0,06$ \\
\hline Métiers tendus fluides & $-0,04 * * *$ & $-0,12$ & $0,28 * * *$ & $0,05 * * *$ & $-0,10 * *$ \\
\hline Métiers tendus difficiles & $-0,03 * *$ & $-0,06$ & 0,04 & 0,02 & $-0,06$ \\
\hline Métiers détendus faciles (réf.) & & & & & \\
\hline
\end{tabular}

Notes : ne sont ici présentés que les résultats concernant l'appartenance à une classe donnée de la typologie. Les modèles estimés sont des probit simples avec calcul d'effets marginaux sauf dans le cas du nombre de personnes impliquées où il s'agit d'un probit multinomial avec 2 personnes impliquées dans le recrutement comme référence et du coût interne pour lequel il s'agit d'un probit ordonné (moins d'1 jour, 1 à 2 jours, 3 jours à 1 semaine, plus d' 1 semaine). Ces modèles comprennent également les variables de contrôle citées en note du tableau 2. Les symboles *,** et *** représentent les seuils de significativité statistique à 10,5 et $1 \%$.

Lecture: le fait d'appartenir à la classe des «métiers tendus fluides » plutôt qu'à celle des «métiers détendus faciles » diminue de 4 points de \% la probabilité que l'établissement dispose d'un service des ressources humaines.

Champ : recrutements des établissements d'au moins un salarié du secteur concurrentiel ayant nouvellement recruté en CDI ou en CDD de plus d'un mois entre septembre et novembre hors domaines professionnels A (agriculture, marine, pêche), P (administration publique, professions juridiques, armée et police) et X (politique, religion); France métropolitaine.

Source : Dares, enquête Ofer 2016

n'y ait qu'une seule personne impliquée n'y est probablement pas étranger mais n'est pas le seul élément explicatif. Les employeurs de ces deux classes ont également plus souvent demandé aux candidats de remplir un dossier de candidature, ce qui a pu contribuer à faciliter le tri des candidatures.

Quelles concessions les recruteurs sont-ils prêts à faire selon le contexte dans lequel ils recrutent? On pourrait s'attendre à ce que les recruteurs intervenant dans un contexte global de tension élevée soient disposés, ex ante, à davantage de concessions que les autres. Or nos résultats ne vont pas dans ce sens. Ainsi, bien qu'ils interviennent dans un contexte global de tension faible, les employeurs de la classe des «métiers très détendus » sont les plus disposés à faire des concessions sur les caractéristiques de la personne recrutée. En effet, ils ont moins d'attentes sur le niveau de diplôme que ceux de la classe des «métiers détendus faciles ${ }^{23}$ et sont davantage disposés à arbitrer entre diplôme et expérience que ceux des autres classes ${ }^{24}$ (tableau 7). $E x$ post, en cas de difficultés, ils ont d'ailleurs davantage de chances d'avoir modifié les caractéristiques du poste à pourvoir que les employeurs des autres classes de métiers, à l'exception de celle des « métiers tendus difficiles », même s'ils n' ont pas pour autant réduit leurs exigences vis-à-vis des candidats. Ils sont également plus nombreux à avoir recruté un chômeur, cela pouvant en partie s'expliquer par le fait qu'ils aient privilégié le SPE lors de leur recherche de candidats. Les recruteurs de la classe des

23. De plus, parmi ceux ayant une attente vis-à-vis du diplôme, les recruteurs de cette classe sont plus nombreux à avoir recruté une personne ayant un niveau inférieur à celui attendu.

24. Plus précisément, ils sont plus nombreux à déclarer qu'ils auraient pu embaucher quelqu'un de moins diplômé mais de plus expérimenté ou, inversement, qu'ils auraient pu embaucher quelqu'un de moins expérimenté mais de plus diplômé. 
TABLEAU 7 - Attentes et arbitrages potentiels des recruteurs selon les cinq classes de métiers

\begin{tabular}{lccccc}
\hline & $\begin{array}{c}\text { Aucune } \\
\text { attente en } \\
\text { matière de } \\
\text { diplôme }\end{array}$ & $\begin{array}{c}\text { Arbitrage } \\
\text { diplôme- } \\
\text { expérience }\end{array}$ & $\begin{array}{c}\text { Arbitrage } \\
\text { expérience- } \\
\text { diplôme }\end{array}$ & $\begin{array}{c}\text { Embauche possible d'un chômeur } \\
\text { à compétences égales (3) }\end{array}$ \\
\cline { 5 - 6 } & & & & $\begin{array}{c}\text { À condition que son } \\
\text { épisode de chômage ne } \\
\text { soit pas trop long }\end{array}$ & Non \\
Métiers très détendus & $0,11 * * *$ & $0,08 * * *$ & $0,03 *$ & $-0,06$ & $-0,14$ \\
Métiers concurrentiels & $-0,01$ & 0,02 & $-0,01$ & 0,11 & $-0,05$ \\
Métiers tendus fluides & $0,03 *$ & $-0,07 * * *$ & 0,01 & $0,27 * * *$ & 0,07 \\
Métiers tendus difficiles & $-0,03 *$ & $-0,02$ & $-0,02$ & $0,30 * * *$ & 0,12 \\
Métiers détendus faciles (réf.) & & & & & \\
\hline
\end{tabular}

Notes : plus précisément, pour (1), la question était «Auriez-vous pu embaucher quelqu'un qui soit moins diplômé mais plus expérimenté ? » et pour (2), «Auriez-vous pu embaucher quelqu'un qui soit moins expérimenté mais plus diplômé? ». Pour (3), la question était, sur le champ des employeurs n'ayant pas recruté un chômeur, « À compétences égales, auriez-vous embauché un chômeur? » et la situation de référence est « Oui, quelle que soit la durée de son épisode de chômage ».

Ne sont ici présentés que les résultats concernant l'appartenance à une classe donnée de la typologie. Les modèles estimés sont des probit simples avec calcul d'effets marginaux sauf dans le cas de l'embauche d'un chômeur où il s'agit d'un probit multinomial avec l'embauche d'un chômeur quelle que soit la durée de son épisode de chômage comme référence. Ces modèles comprennent également les variables de contrôle citées en note du tableau 2. Les symboles *,** et *** représentent les seuils de significativité statistique à 10,5 et $1 \%$. Lecture : le fait d'appartenir à la classe des «métiers très détendus » plutôt qu'à celle des «métiers détendus faciles » augmente de 11 points de \% la probabilité de déclarer n'avoir aucune attente en matière de diplôme.

Champ : recrutements des établissements d'au moins un salarié du secteur concurrentiel ayant nouvellement recruté en CDI ou en CDD de plus d'un mois entre septembre et novembre 2015 hors domaines professionnels A (agriculture, marine, pêche), P (administration publique, professions juridiques, armée et police) et X (politique, religion); France métropolitaine.

Source: Dares, enquête Ofer 2016.

« métiers tendus fluides » avaient également moins d'attentes par rapport au diplôme, mais n'étaient en revanche pas plus souvent disposés que les recruteurs des autres classes de métiers à réduire le niveau de ce dernier en contrepartie d'une expérience supplémentaire du candidat ${ }^{25}$. De leur côté, les employeurs de la classe des «métiers tendus difficiles » avaient davantage d'attentes en matière de diplôme. Comme ceux de la classe des «métiers tendus fluides », les recruteurs de cette classe n'ayant pas embauché un chômeur se déclarent plus souvent prêts à en recruter un, mais à condition que sa durée de chômage n' ait pas été trop longue. Les employeurs de cette classe sont également moins nombreux à avoir effectivement recruté un chômeur tout comme ceux de la classe des «métiers concurrentiels ». Les recruteurs confrontés aux contextes globaux de tension les plus difficiles ne semblent ainsi pas davantage disposés à faire des concessions sur les compétences ou la situation antérieure de la personne recrutée. Néanmoins, lorsqu'ils déclarent avoir été confrontés à des difficultés, les employeurs de la classe des «métiers tendus difficiles » sont plus nombreux à mentionner avoir modifié les caractéristiques de leur poste pour faciliter le recrutement.

Au moment de dresser le bilan du recrutement réalisé, ce sont les recruteurs de la classe des « métiers tendus concurrentiels » qui sont les plus satisfaits de la personne

25. Cela peut s'expliquer par le fait que les métiers concernés, majoritairement dans les secteurs du commerce et de l'hébergement et restauration, nécessitent davantage des compétences relationnelles - qualités personnelles - que des compétences techniques qui s'acquièrent par diplôme ou expérience professionnelle. 
embauchée: ils déclarent plus souvent que les autres qu'ils recruteraient la même personne si l'embauche était à refaire et que le recrutement a répondu à leurs principaux critères de sélection. L'absence plus fréquente d'un service RH et leur manque d'habitude de recruter ne semblent pas leur avoir compliqué la tâche. A contrario, les recruteurs de la classe des «métiers tendus fluides » font plus souvent état d'une rupture prématurée de la relation d'emploi, que cette dernière soit de leur fait ou de celui de la personne embauchée. Le déficit de candidatures a peut-être eu pour effet de réduire leurs exigences à l'égard des candidats, mais également la pérennité de la relation d'emploi ainsi nouée.

Enfin, les efforts de recrutement des employeurs ont-ils permis de limiter leurs difficultés d'embauche au regard du contexte global de tension auquel ils sont confrontés? D'après nos résultats, cela semble partiellement être le cas. Ainsi, nombreux à anticiper des difficultés de recrutement et confrontés à un indicateur de tension élevé, les recruteurs de la classe des «métiers tendus fluides » n'en déclarent pas davantage que les autres ex post. Leur processus de recrutement s'est avéré moins long que dans les autres classes, à l'exception de celle des « métiers très détendus », probablement en raison de leur mobilisation plus fréquente des candidatures spontanées, un canal rapide et peu coûteux à activer, et de l'implication d'une seule personne dans le recrutement. Leur investissement dans le recrutement a toutefois été conséquent puisqu'ils ont demandé un nombre plus élevé de pièces aux candidats, en ont reçu une plus grande part en entretien individuel et le chef d'établissement a plus souvent participé à l'embauche que ce que l'on observe dans la classe des «métiers détendus faciles».

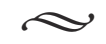

Pour étudier les liens entre le contexte du marché du travail, les difficultés de recrutement et les efforts de recrutement des employeurs, nous avons construit une typologie des métiers selon le contexte global de tension évalué par quatre indicateurs: la tension proprement dite, le nombre d'emplois vacants par salarié, le taux de sortie du chômage et les difficultés anticipées de recrutement. Cet indicateur original de contexte global de tension permet d'enrichir l'analyse par rapport à l'indicateur de tension du marché du travail retenu traditionnellement. Parmi les cinq classes de métiers ainsi identifiées, deux classes, représentant environ un quart des recrutements, associent les niveaux de tension et de difficultés anticipées les plus élevés: celles des «métiers tendus difficiles » et des «métiers tendus fluides », la seconde étant caractérisée par un taux de sortie du chômage plus conséquent. Une troisième classe se situe dans une position intermédiaire caractérisée par une forte concurrence sur les postes vacants et un niveau de tension et de difficulté dans la moyenne: celle des «métiers concurrentiels » (concernant près de $20 \%$ des embauches).

Le premier résultat de notre analyse peut être résumé comme suit: embaucher dans un contexte global de tension élevée n'est pas systématiquement synonyme de difficultés de recrutement ex post. Des deux classes couplant tension et difficultés de 
recrutement ex ante, seule celle des «métiers tendus difficiles » se caractérise par un niveau plus élevé de difficultés ex post que celle des «métiers détendus faciles », mise en référence. Cela n'est pas si surprenant car d'autres facteurs que la situation sur le marché du travail peuvent compliquer le processus d'embauche. La corrélation positive entre contexte global de tension élevée et difficultés de recrutement se vérifie pour certaines catégories de difficultés: recruter sur un métier en tension se traduit bien par une pénurie accrue de candidatures, un déficit d'image de l'entreprise, du secteur d'activité ou du métier proposé plus marqué et, dans certains cas, une plus forte concurrence de la part des autres employeurs.

Le deuxième résultat est que la relation positive attendue au vu de la littérature (GAVAZZA et al., 2018) entre tension sur le marché du travail et effort de recrutement ne se concrétise pas pour toutes les dimensions de cet effort. En particulier, un contexte global de tension élevée ne se traduit pas par l'activation d'un plus grand nombre de canaux de recherche de candidats. En revanche, les recruteurs choisissent des canaux spécifiques - un constat déjà réalisé par les travaux économiques sur les canaux - selon le contexte global de tension sur lequel ils sont amenés à embaucher: ils privilégient ainsi les intermédiaires autres que le SPE et leurs relations lorsqu'ils doivent faire face à une forte concurrence sur leurs postes vacants; ils font davantage appel aux candidatures spontanées lorsque le marché du travail est tendu mais plus fluide. In fine, en cas de difficultés, les employeurs recrutant sur un métier en tension déclarent plus souvent avoir intensifié leur effort de recrutement.

Le constat est également mitigé concernant la phase de sélection des candidatures. D'un côté, le recrutement sur un marché du travail tendu s'accompagne de davantage d'efforts mesurés par le nombre de pièces demandées (références, copies de diplôme, $\mathrm{CV}$, lettre de motivation, etc.) et la part des candidats reçus en entretien individuel. D'un autre côté, les employeurs intervenant dans des contextes globaux de tension difficiles ne se distinguent pas par la mobilisation de méthodes de sélection en plus grand nombre ou par une plus grande propension à faire des concessions sur les compétences ou le parcours de la personne recrutée. Par ailleurs, l'équipement et la compétence de ces recruteurs pour embaucher semblent également moindres : ils disposent moins souvent d'un service de RH et, pour certains, ont moins l'habitude de recruter. Marc-Antoine ESTRADE (2013) avait d'ailleurs mis en évidence un risque d'échec de recrutement accru dans les établissements n'ayant pas l'habitude de recruter.

Cet article nous a permis de mettre en regard tension sur le marché du travail, difficultés ex post et efforts de recrutement au niveau du métier; elle présente néanmoins un certain nombre de limites. L'année 2015 est une année particulière au regard des difficultés de recrutement puisqu'elle correspond au niveau le plus bas observé au cours des dix dernières années. De plus, notre mesure des indicateurs de «tension » est réalisée au niveau national et ne permet ainsi pas de caractériser le contexte local du marché du travail. Or ce dernier peut jouer un rôle non négligeable pour certaines catégories de métiers. 


\section{BIBLIOGRAPHIE}

BAgein G., Poujouly C. (2017), « Les sortants des listes de demandeurs d'emploi inscrits à Pôle emploi, en septembre 2016 », Dares indicateurs, n 026.

BaILly F., LÉNÉ A. (2015), «Post-face: retour sur le concept de compétences non académiques », Formation emploi, n ${ }^{\circ} 130$, pp. 69-78.

Bergeat M. (2017), « Les tensions sur le marché du travail au $2^{e}$ trimestre 2017 », Dares indicateurs, $\mathrm{n}^{\circ} 056$.

BERgEAT M., RÉMY V. (2017), «Comment les employeurs recrutent-ils leurs salariés? », Dares analyses, $\mathrm{n}^{\circ} 064$.

BERGEAT M., RÉMY V. (2019), « Les canaux de recrutement ont-ils un effet sur la qualité et le coût des appariements sur le marché du travail français? », Revue économique, vol. 70, $\mathrm{n}^{\circ} 5$, p. 717-749.

BESSY C., LARQUIER G. DE (2010), « Diversité et efficacité des intermédiaires du placement », Revue française d'économie, vol. 25, n² 2, p. 227-270.

Bessy C., Marchal E. (2007), «L'usage des canaux de recrutement par les entreprises », Document de travail, $\mathrm{n}^{\circ} 89$, Centre d'études de l'emploi.

BLACHE G., BUCHNER D. (2015), « Les employeurs entrevoient une amélioration de leurs perspectives d'embauche pour 2015 et de moindres difficultés à recruter », Éclairages et Synthèses, $\mathrm{n}^{\mathrm{o}}$ 11, Pôle emploi.

BLACHe G., GAumont S., ZANDA J.-L. (2016), «Les offres d'emplois difficiles à satisfaire: les difficultés sont d'origines multiples et se concentrent sur certains secteurs », Éclairages et Synthèses, $\mathrm{n}^{\circ} 21$, Pôle emploi.

Conseil D'orientation pour L'EMPLOI (COE) (2013), Emplois durablement vacants et difficultés de recrutement, septembre.

Davis S. J., Faberman R. J., Haltiwanger J. C. (2013), “The Establishment-Level Behavior of Vacancies and Hiring", The Quarterly Journal of Economics, vol. 128, n 2, pp. 581-622.

DeVAro J. (2005), "Employer Recruitment Strategies and the Labor Market Outcomes of New Hires", Economic Inquiry, vol. 43, n 2 2, pp. 263-282.

DeVAro J. (2008), "The Labor Market Effects of Employer Recruitment Choice", European Economic Review, vol. 52, n 2, pp. 283-314.

ESTRADE M.-A. (2013), « Les emplois non pourvus: mythes et réalités », Regards croisés sur l'économie, $\mathrm{n}^{\circ}$ 1, pp. 151-167.

FABERMAn R. J. (2014), "Recruiting Intensity”, IZA World of Labor [en ligne]. https://doi. org/10.15185/izawol.21

FonDEUR Y. (2013), «Services de conseil en informatique: recruter pour placer », La Revue de l'Ires, $\mathrm{n}^{\circ}$ 76, pp. 99-125.

FONDEUR Y., ZANDA J.-L. (2009), « Les emplois “vacants” », Connaissance de l'emploi, n 64, Centre d'études de l'emploi. 
ForTÉ M., Monchatre S. (2013), « Recruter dans l'hôtellerie-restauration : quelle sélectivité sur un marché du travail en tension? », La Revue de l'Ires, $\mathrm{n}^{\circ}$ 76, pp. 127-150.

Gavazza A., Mongey S., Violante G. L. (2018), “Aggregate Recruiting Intensity”, American Economic Review, vol. 108, n 8, pp. 2088-2127.

Gorter C., NijkAmp P., Rietveld P. (1996), “Employers’ Recruitment Behaviour and Vacancy Duration: An Empirical Analysis for the Dutch Labour Market", Applied Economics, vol. 28, $\mathrm{n}^{\mathrm{o}} 11$, pp. 1463-1474.

Herz B., van Rens T. (2018), “Accounting for Mismatch Unemployment”, Londres, Centre for Economic Policy Research.

INSEE (2017), «Que nous disent les entreprises sur les barrières à l'embauche? », Note de conjoncture, juin, pp. 74-78.

Jaudeau C., Jousselin E., Roux S., Verdugo G. (2015), « Les entreprises dans la crise : premiers résultats d'une enquête européenne », Bulletin de la Banque de France, n 201, pp. 33-40.

LhommeAu B., RÉMY V. (2019a), « Comment les employeurs surmontent-ils leurs difficultés de recrutement? », Dares analyses, $\mathrm{n}^{\circ} 032$.

LHOMMEAU B., RÉmY V. (2019b), « Le recrutement n'est pas toujours un long fleuve tranquille. $17 \%$ des recrutements sont jugés difficiles par les recruteurs », Dares analyses, nº 031.

Marsden P. V. (1988), "Homogeneity in Confiding Relations", Social Networks, vol. 10, n 1, pp. 57-76.

Marsden P. V., Gorman E. H. (2001), "Social Networks, Job Changes and Recruitment”, in Berg I., Kalleberg A. (eds), Sourcebook of Labor Markets: Evolving Structures and Processes, New York, Springer, pp. 467-502.

Niang M., Vroylandt T. (2020), «Les tensions sur le marché du travail en 2019 », Dares résultats, $\mathrm{n}^{\circ} 032$.

PISSARIDEs C. A. (2000), Equilibrium Unemployment Theory, Cambridge (Mass.), MIT Press.

REES A. (1966), "Information Networks in Labor Markets", American Economic Review, vol. $56, \mathrm{n}^{\mathrm{o}} 1 / 2$, pp. 559-566.

Rees A., Shultz G. P. (1970), Workers and Wages in an Urban Labor Market, Chicago, University of Chicago Press.

RieuCAU G., SALOGNON M. (2013), « Le recrutement dans la grande distribution: des pratiques ajustées? », La Revue de l'Ires, nº 76, pp. 45-69.

RoPer S. (1988), "Recruitment Methods and Vacancy Duration", Scottish Journal of Political Economy, vol. 35, n 1, pp. 51-64.

Russo G., Gorter C., NiJkamp P., Rietveld P. (1997), “Employers' Recruitment Behaviour: An Empirical Analysis of the Role of Personnel Management Attitudes", Labour, vol. 11, n 3, pp. 599-623.

Russo G., Rietveld P., NiJKAMP P., GorTer C. (2000), "Recruitment Channel Use and Applicant Arrival: An Empirical Analysis", Empirical Economics, vol. 25, n 4, pp. 673-697. 
Quels liens entre tension sur le marché du travail, difficultés de recrutement et efforts de recherche des employeurs?

SABATIER M. (2010), "Filling Vacancies: Identifying the Most Efficient Recruitment Channel", Economics Bulletin, vol. 30, n 4, pp. 3355-3368.

Şahin A., Song J., Topa G., Violante G. L. (2014), "Mismatch Unemployment", American Economic Review, vol. 104, n 11 , pp. 3529-3564. 


\section{ANNEXE}

TABLEAU 1A - Caractéristiques des établissements et des postes selon les cinq classes de métiers

\begin{tabular}{|c|c|c|c|c|c|c|}
\hline & $\begin{array}{l}\text { Métiers } \\
\text { détendus } \\
\text { faciles }\end{array}$ & $\begin{array}{l}\text { Métiers } \\
\text { très } \\
\text { détendus }\end{array}$ & $\begin{array}{l}\text { Métiers } \\
\text { concur- } \\
\text { rentiels }\end{array}$ & $\begin{array}{l}\text { Métiers } \\
\text { tendus } \\
\text { fluides }\end{array}$ & $\begin{array}{c}\text { Métiers } \\
\text { tendus } \\
\text { difficiles }\end{array}$ & Ensemble \\
\hline \multicolumn{7}{|l|}{ Caractéristiques de l'établissement } \\
\hline \multicolumn{7}{|l|}{$\begin{array}{l}\text { Secteur d'activité } \\
\end{array}$} \\
\hline Industrie & 11 & 10 & 8 & 4 & 29 & 11 \\
\hline Construction & 12 & 2 & 8 & 2 & 17 & 9 \\
\hline Commerce & 28 & 12 & 11 & 17 & 12 & 19 \\
\hline Transports & 10 & 5 & 1 & 0 & 2 & 5 \\
\hline Hébergement et restauration & 3 & 4 & 19 & 41 & 1 & 11 \\
\hline Information et communication & 3 & 1 & 4 & 1 & 13 & 4 \\
\hline Services financiers et immobiliers & 4 & 3 & 3 & 7 & 0 & 4 \\
\hline Services aux entreprises & 14 & 28 & 10 & 7 & 17 & 15 \\
\hline Services non marchands & 11 & 28 & 24 & 10 & 7 & 16 \\
\hline Services aux ménages & 3 & 7 & 12 & 9 & 2 & 6 \\
\hline \multicolumn{7}{|l|}{ Taille } \\
\hline Moins de 10 salariés & 33 & 22 & 38 & 42 & 36 & 33 \\
\hline De 10 à 49 salariés & 32 & 36 & 34 & 34 & 24 & 32 \\
\hline De 50 à 199 salariés & 18 & 23 & 15 & 17 & 18 & 18 \\
\hline 200 salariés ou plus & 17 & 18 & 14 & 8 & 23 & 16 \\
\hline Appartient à un groupe & 45 & 37 & 34 & 39 & 39 & 40 \\
\hline $\begin{array}{l}\text { Fait partie d'une entreprise } \\
\text { multi-établissements }\end{array}$ & 49 & 46 & 39 & 38 & 42 & 44 \\
\hline \multicolumn{7}{|l|}{ Ancienneté de l'établissement } \\
\hline Inférieure à 5 ans & 37 & 35 & 34 & 40 & 37 & 37 \\
\hline De 5 à 10 ans & 22 & 23 & 19 & 18 & 22 & 21 \\
\hline Supérieure à 10 ans & 41 & 42 & 47 & 42 & 41 & 42 \\
\hline $\begin{array}{l}\text { Situé dans une commune appartenant } \\
\text { à l'aire urbaine de Paris }\end{array}$ & 32 & 28 & 34 & 31 & 31 & 32 \\
\hline Recours à l'intérim en 2015 & 13 & 11 & 11 & 7 & 20 & 12 \\
\hline Recours aux contrats courts en 2015 & 3 & 34 & 25 & 22 & 17 & 27 \\
\hline \multicolumn{7}{|l|}{$\begin{array}{l}\text { Introduction au cours des trois } \\
\text { dernières années (1) }\end{array}$} \\
\hline de nouveaux produits ou services & 52 & 38 & 45 & 56 & 55 & 49 \\
\hline de nouvelles techniques & 37 & 37 & 34 & 38 & 45 & 38 \\
\hline d'une nouvelle organisation du travail & 39 & 39 & 33 & 40 & 39 & 38 \\
\hline $\begin{array}{l}\text { Plusieurs recrutements sur le même } \\
\text { type de poste }\end{array}$ & 32 & 35 & 28 & 32 & 28 & 32 \\
\hline
\end{tabular}


Quels liens entre tension sur le marché du travail, difficultés de recrutement

et efforts de recherche des employeurs?

\begin{tabular}{lcccccc}
\hline & $\begin{array}{c}\text { Métiers } \\
\text { détendus } \\
\text { faciles }\end{array}$ & $\begin{array}{c}\text { Métiers } \\
\text { très } \\
\text { détendus }\end{array}$ & $\begin{array}{c}\text { Métiers } \\
\text { concur- } \\
\text { rentiels }\end{array}$ & $\begin{array}{c}\text { Métiers } \\
\text { tendus } \\
\text { fluides }\end{array}$ & $\begin{array}{c}\text { Métiers } \\
\text { tendus } \\
\text { difficiles }\end{array}$ & $\begin{array}{c}\text { Ensemble } \\
\text { Caractéristiques du poste }\end{array}$ \\
\hline Création de poste & 52 & 52 & 47 & 35 & 62 & 50 \\
\hline Type de contrat & & & & & & \\
CDD court (de 1 à 3 mois) & 17 & 19 & 11 & 12 & 10 & 15 \\
CDI & 44 & 48 & 49 & 60 & 46 & 48 \\
CDD long (3 mois ou plus) & 39 & 33 & 40 & 28 & 44 & 37 \\
\hline Contrat à temps partiel & 28 & 47 & 36 & 33 & 11 & 32 \\
\hline Contrat assorti d'une aide financière & 17 & 19 & 14 & 15 & 22 & 17 \\
\hline Catégorie socioprofessionnelle & & & & & & \\
Cadre & 7 & 1 & 26 & 2 & 22 & 11 \\
Profession intermédiaire & 9 & 19 & 32 & 38 & 40 & 23 \\
Employé non qualifié & 29 & 38 & 11 & 40 & 0 & 26 \\
Employé qualifié & 23 & 0 & 7 & 16 & 0 & 12 \\
Ouvrier non qualifié & 14 & 35 & 7 & 1 & 9 & 14 \\
Ouvrier qualifié & 17 & 7 & 18 & 3 & 28 & 15 \\
\hline
\end{tabular}

Note: (1) Sur le champ de ceux ayant au moins trois ans d'ancienneté.

Lecture : $10 \%$ des recrutements des établissements de la classe des «métiers très détendus » ont eu lieu dans le secteur de l'industrie. Champ : recrutements des établissements d'au moins un salarié du secteur concurrentiel ayant nouvellement recruté en CDI ou en CDD de plus d'un mois entre septembre et novembre 2015 hors domaines professionnels A (agriculture, marine, pêche), P (administration publique, professions juridiques, armée et police) et X (politique, religion); France métropolitaine.

Source : Dares, enquête Ofer 2016.

\section{TABLEAU 1B - Indicateurs complémentaires de conditions de travail selon les cinq classes de métiers}

\begin{tabular}{|c|c|c|c|c|c|c|}
\hline & $\begin{array}{l}\text { Métiers } \\
\text { détendus } \\
\text { faciles }\end{array}$ & $\begin{array}{l}\text { Métiers } \\
\text { très } \\
\text { détendus }\end{array}$ & $\begin{array}{l}\text { Métiers } \\
\text { concur- } \\
\text { rentiels }\end{array}$ & $\begin{array}{l}\text { Métiers } \\
\text { tendus } \\
\text { fluides }\end{array}$ & $\begin{array}{c}\text { Métiers } \\
\text { tendus } \\
\text { difficiles }\end{array}$ & Ensemble \\
\hline \multicolumn{7}{|l|}{ Part de salariés } \\
\hline $\begin{array}{l}\text { ayant le sentiment de devoir changer } \\
\text { de métier dans les } 3 \text { prochaines années }\end{array}$ & 35 & 33 & 33 & 29 & 26 & 32 \\
\hline $\begin{array}{l}\text { rencontrant au moins } 3 \text { contraintes } \\
\text { physiques (1) }\end{array}$ & 30 & 41 & 29 & 33 & 42 & 35 \\
\hline effectuant des tâches répétitives & 46 & 58 & 33 & 42 & 36 & 44 \\
\hline $\begin{array}{l}\text { travaillant régulièrement avec } \\
\text { des horaires atypiques }\end{array}$ & 44 & 39 & 45 & 37 & 50 & 43 \\
\hline $\begin{array}{l}\text { souhaitant transmettre leur métier } \\
\text { à leurs enfants }\end{array}$ & 36 & 23 & 39 & 37 & 40 & 35 \\
\hline
\end{tabular}

Notes : les métiers sont pondérés par les effectifs salariés.

(1) L'emploi doit comporter au moins trois contraintes parmi les cinq suivantes : porter ou déplacer des charges lourdes, rester longtemps debout, rester longtemps dans une autre posture pénible ou fatigante à la longue, effectuer des déplacements à pied longs ou fréquents, subir des secousses ou vibrations.

Lecture : la part des salariés ayant le sentiment de devoir changer de métier dans les trois prochaines années est de $33 \%$ dans la classe des «métiers très détendus ».

Champ : emploi salarié, hors domaines professionnels A (agriculture, marine, pêche), $\mathrm{P}$ (administration publique, professions juridiques, armée et police) et $\mathrm{X}$ (politique, religion); France métropolitaine.

Sources: Insee, enquêtes Emploi 2014, 2015 et 2016; Pôle emploi et Dares, STMT; Pôle emploi, enquête Besoins en main-d'œuvre; Dares-Drees-DGAFP, enquête Conditions de travail - risques psychosociaux 2016. 
